C. Bonnafé, G. I. Lehrer and J. Michel

Nagoya Math. J.

Vol. 182 (2006), 135-170

\title{
TWISTED INVARIANT THEORY FOR REFLECTION GROUPS
}

\author{
C. BONNAFÉ, G. I. LEHRER AND J. MICHEL \\ Dedicated to George Lusztig on his 60th birthday
}

\begin{abstract}
Let $G$ be a finite reflection group acting in a complex vector space $V=\mathbb{C}^{r}$, whose coordinate ring will be denoted by $S$. Any element $\gamma \in \operatorname{GL}(V)$ which normalises $G$ acts on the $\operatorname{ring} S^{G}$ of $G$-invariants. We attach invariants of the coset $G \gamma$ to this action, and show that if $G^{\prime}$ is a parabolic subgroup of $G$, also normalised by $\gamma$, the invariants attaching to $G^{\prime} \gamma$ are essentially the same as those of $G \gamma$. Four applications are given. First, we give a generalisation of a result of Springer-Stembridge which relates the module structures of the coinvariant algebras of $G$ and $G^{\prime}$ and secondly, we give a general criterion for an element of $G \gamma$ to be regular (in Springer's sense) in invariant-theoretic terms, and use it to prove that up to a central element, all reflection cosets contain a regular element. Third, we prove the existence in any well-generated group, of analogues of Coxeter elements of the real reflection groups. Finally, we apply the analysis to quotients of $G$ which are themselves reflection groups.
\end{abstract}

\section{$\S 1$. Introduction}

Let $G$ be a finite group generated by (pseudo)reflections in a vector space of dimension $r<\infty$ over the algebraically closed field $K$ of characteristic zero. The purpose of this work is to discuss the "twisted case" of various phenomena associated with the structure and invariant theory of $G$. That is, we take an element $\gamma \in \mathrm{GL}(V)$ which normalises $G$, and consider how it acts on the invariants and covariants (for various representations) of $G$, and properties of its eigenspaces. In particular we study generalisations of the results of [LS1] and [LM], and the action of $\langle G, \gamma\rangle$ on covariants. Our basic method is a variation on the theme of [L], which enables us to relate various sets of constants associated to $G$ with the corresponding ones for

Received July 4, 2005.

Revised February 16, 2006.

2000 Mathematics Subject Classification: Primary 20F55; Secondary 20H15, 51F15. 
parabolic subgroups. In many cases, we identify the relevant constants as eigenvalues of certain transformations.

Specifically, to any finite dimensional $\langle G, \gamma\rangle$-module $M$ we associate a (multi)set of $m=\operatorname{dim} M$ constants $\varepsilon_{\iota}(M)$, which depend only on the coset $G \gamma$. Various algebraic and geometric properties of $G \gamma$ may then be expressed in terms of the constants $\varepsilon_{\iota}$. For example the condition that $\zeta \in K^{\times}$be regular for $G \gamma$ may be expressed in terms of the $\varepsilon_{\iota}(V)$ and $\varepsilon_{\iota}\left(V^{*}\right)$ (see Section 5 below). A theme of this work is that if $G^{\prime}$ is a parabolic subgroup of $G$ which is normalised by $\gamma$, then the constants $\varepsilon_{\iota}(M)$ are the same whether $M$ is regarded as a module for $\langle G, \gamma\rangle$ or $\left\langle G^{\prime}, \gamma\right\rangle$ (see (4.4) below. This idea is behind many of the results and their proofs.

One set of applications of our results is to the question of regular elements and regular eigenvalues for reflection cosets. The vector $v \in V$ is $(G$-)regular if $v$ does not lie on any reflecting hyperplane of $G$. The element $\gamma \in \gamma G$ is regular if it has a regular eigenvector; if $\gamma v=\zeta v$, then $\zeta$ is called a regular eigenvalue and its order (when $\gamma$ has finite order) is a regular number. In this work we give precise criteria for an eigenvalue to be regular for a coset, and apply these to various questions. When $\gamma \in G$, it is trivial that the identity element of $G$ is a regular element, with corresponding eigenvalue 1. In general, it is not even obvious that any regular elements exist. We show that, with obviously necessary qualifications, they do ${ }^{1}$.

In the case of "well-generated groups" we use our criterion for regularity which is couched in terms of the $\varepsilon_{\iota}$ to produce a twisted analogue of Coxeter elements of a real reflection group, and a twisted analogue for a reflection coset of the Coxeter number of a real reflection group.

Another significant application of our results concerning parabolic subgroups is to the module structure of the coinvariant algebra for the group $\langle G, \Gamma\rangle$, where $\Gamma$ is a finite subgroup of the normaliser of $G$ in $\operatorname{GL}(V)$. Our result, Theorem 4.6, generalises one of Stembridge (in the untwisted regular case), whose proof goes back to Springer's computation of the eigenvalues of a regular element in any representation. One interpretation of Stembridge's result is that it gives an expression for the $G$-module structure of the sum of certain graded components of the coinvariant algebra. Our result generalises this in two ways; first, by considering a larger class of sums by removing the restriction of regularity, and second, by considering

\footnotetext{
${ }^{1}$ We have recently discovered that this result also appears in the work [Ma] of G. Malle, whom we thank for a preprint. Our proof involves less case by case checking.
} 
the twisted structure of the coinvariant algebra. The statement in (4.6) expresses the sum of certain graded components of the coinvariant algebra of $G$ as a representation induced from the coinvariant algebra of a parabolic subgroup.

In the final section, we explore the relationship between our twisted invariants and the reflection quotients of reflection groups studied in [BBR]. These are quotients $G / L$ of $G$ which act as reflection groups on the tangent space at 0 of $V / L$, where $L$ is a normal subgroup of $G$. Here we are able to relate the constants and other invariants of $G$ with those of its reflection quotients in the above sense.

\section{§2. Background and notation}

Let $K$ be an algebraically closed field of characteristic 0 , and $V$ be a $K$-vector space of dimension $r$. Let $G \subset \mathrm{GL}(V)$ be a finite subgroup generated by (pseudo)reflections. Denote by $\mathcal{A}$ (or $\mathcal{A}(G)$ when appropriate) the corresponding set of reflecting hyperplanes, and for each $H \in \mathcal{A}$ choose a linear form $L_{H} \in V^{*}$ with kernel $H$. Let $S$ be the algebra of polynomial functions on $V$; it may be identified with the symmetric algebra of the dual vector space $V^{*}$. The subalgebra $S^{G}$ of $G$-invariant functions is a polynomial algebra. Let $\mathcal{N}$ be the normaliser of $G$ in $\operatorname{GL}(V)$; this is a (not necessarily connected) reductive group. Denote by $I$ the ideal of $S$ generated by elements of $S^{G}$ with no constant term, and let $\mathcal{H}$ be the space of $G$-harmonic polynomials, i.e., the polynomials which are annihilated by all $G$-invariant polynomial differential operators on $S$ with no constant term. Then $\mathcal{H}$ is $\mathcal{N}$-stable and $I \oplus \mathcal{H}=S$. So, by Chevalley's theorem, the natural map $S^{G} \otimes \mathcal{H} \rightarrow S$ is an isomorphism of $\mathcal{N}$-modules. The algebra $S_{G}=S / I$ is called the algebra of coinvariants. Again by a result of Chevalley, it is isomorphic as a $G$-module to $K[G]$, so that $\mathcal{H}$ is also a $G$-submodule of $S$, isomorphic to the regular representation of $G$, which is stable under $\mathcal{N}$.

Let $M$ be any finite dimensional $G$-module; then $\left(S \otimes M^{*}\right)^{G} \simeq S^{G} \otimes(\mathcal{H} \otimes$ $\left.M^{*}\right)^{G}$ is free as $S^{G}$-module, and since $\mathcal{H}$ realises the regular representation of $G$, it is of rank $m=\operatorname{dim} M$. Notice that $S \otimes M^{*}$ is graded: we declare $\operatorname{deg} F \otimes y=\operatorname{deg} F$, for $F$ a homogeneous element of $S$ and $y \in M^{*}$. If $u_{1}, \ldots, u_{m}$ is a homogeneous linear basis of $\left(\mathcal{H} \otimes M^{*}\right)^{G}$, it is clearly an $S^{G_{-}}$ basis of $\left(S \otimes M^{*}\right)^{G}$. The numbers $m_{i}(M):=\operatorname{deg} u_{i}$ are the $M$-exponents of $G$.

The following observations concerning $G$ and $\mathcal{N}$ are useful, and will often be used without comment. 
Remark 2.1. Since $G$ is generated by reflections, there is a unique decomposition $V=V^{G} \oplus V_{1} \oplus \cdots \oplus V_{k}$, where the $V_{i}$ are irreducible, pairwise non-isomorphic, non-trivial $G$-submodules of $V$, and correspondingly $G=G_{1} \times \cdots \times G_{k}$, where $G_{i}$ acts as an irreducible reflection group on $V_{i}$, and acts trivially on the other summands. Of course distinct pairs $\left(G_{i}, V_{i}\right)$ may be isomorphic as reflection groups.

Clearly $C_{\mathrm{GL}(V)}(G) \simeq \mathrm{GL}\left(V^{G}\right) \times K^{\times} \times \cdots \times K^{\times}\left(k\right.$ copies of $\left.K^{\times}\right)$, while $\mathcal{N}=\mathrm{GL}\left(V^{G}\right) \times N_{\mathrm{GL}\left(V_{1} \oplus \cdots \oplus V_{k}\right)}\left(G_{1} \times \cdots \times G_{k}\right)$. Moreover $\mathcal{N} / C_{\mathrm{GL}(V)}(G)$ is evidently a finite group, since it acts faithfully as a group of automorphisms of $G$. Thus if $\gamma \in \mathcal{N}$, there exists $n \geq 1$ such that $\gamma^{n}$ centralises $G$.

Now any element $\gamma \in \mathcal{N}$ is of the form $x \gamma^{\prime}$, where $x \in \mathrm{GL}\left(V^{G}\right) \leq$ $C_{\mathrm{GL}(V)}(G)$ and $\gamma^{\prime} \in N_{\mathrm{GL}\left(V_{1} \oplus \cdots \oplus V_{k}\right)}\left(G_{1} \times \cdots \times G_{k}\right)$. Since $\gamma^{\prime}$ permutes the subspaces $V_{i}$ and $\operatorname{Hom}_{G}\left(V_{i}, V_{j}\right)$ has dimension at most 1, there exists $z^{\prime} \in K^{\times} \times \cdots \times K^{\times}$such that $\gamma^{\prime} z^{\prime}$ has finite order, and taking $z=x z^{\prime}$, we see that for any $\gamma \in \mathcal{N}$ there is an element $z \in C_{\mathrm{GL}(V)}(G)$ such that $z \gamma$ is of finite order. If $G$ is essential, i.e. if $V^{G}=0$, then $x=1$ above, so $z$ is semisimple, and $\mathcal{N}$ is a finite extension of a torus which centralises $G$; in this case, every element of $\mathcal{N}$ is semisimple. In general, since every unipotent element of $\mathcal{N}$ centralises $G$, the action of any element $\gamma \in \mathcal{N}$ on $G$ coincides with the action of its semisimple part on $G$.

Remark 2.1 shows that the action on $G$ of an arbitrary element $\gamma$ of $\mathrm{GL}(V)$ is induced by a semisimple element of finite order, and so no generality is lost by making the

Hypothesis. Henceforth we take $\gamma$ to be a fixed semisimple element of $\mathrm{GL}(V)$, which normalises $G$.

The coset $G \gamma$ will be referred to as a reflection coset. Let $M$ be a $\langle G, \gamma\rangle$-module on which $\gamma$ acts semisimply. We shall define some important constants associated with the coset $G \gamma$.

Since $\gamma$ acts semisimply on $\left(\mathcal{H} \otimes M^{*}\right)^{G}$, the basis elements $u_{i}$ above may be taken to be eigenvectors for $\gamma$. For each such $M$, denote by $\mathcal{B}(M, \gamma)$ a fixed homogeneous basis of $\left(\mathcal{H} \otimes M^{*}\right)^{G}$ which consists of eigenvectors of $\gamma$. Given $\iota \in \mathcal{B}(M, \gamma)$, denote by $\varepsilon_{\iota}(M)$, or $\varepsilon_{\iota}$ when unambiguous, the corresponding eigenvalue of $\gamma$, and by $m_{\iota}(M)=m_{\iota}$ the degree of $\iota$. Thus $\gamma \iota=\varepsilon_{\iota} \iota$ for $\iota \in \mathcal{B}(M, \gamma)$, and for any $g \in G, g \gamma \iota=\varepsilon_{\iota} \iota$, whence the multiset of pairs $\left\{\left(\varepsilon_{\iota}, m_{\iota}\right) \mid \iota \in \mathcal{B}(M, \gamma)\right\}$ depends only on (the isomorphism class of) $M$ and the coset $G \gamma$, and not on the choice of $\gamma \in G \gamma$ or on the basis $\mathcal{B}(M, \gamma)$. 
Definition 2.2. For any $\langle G, \gamma\rangle$-module $M$, the multiset $\left\{\varepsilon_{\iota}(M)=\varepsilon_{\iota} \mid\right.$ $\iota \in \mathcal{B}(M, \gamma)\}$ will be referred to as the multiset of $M$-factors of $G \gamma$.

Remark 2.3. Let $\zeta \in K^{\times}$and let $M$ be a $\left\langle G, \gamma, \zeta \operatorname{Id}_{V}\right\rangle$-module on which $\zeta \operatorname{Id}_{V}$ acts as multiplication by a scalar, say $\zeta_{M}$. Then we may take $\mathcal{B}\left(M, \zeta^{-1} \gamma\right)=\mathcal{B}(M, \gamma)$ and we have $\varepsilon_{\iota}\left(\zeta^{-1} \gamma\right)=\zeta_{M} \zeta^{m_{\iota}} \varepsilon_{\iota}(\gamma)$ for every $\iota \in \mathcal{B}(M, \gamma)$.

The cases $M=V$ and $M=V^{*}$ will figure prominently below. In these cases, for simplicity, we write $\mathcal{B}(\gamma)=\mathcal{B}(V, \gamma), \mathcal{B}^{*}(\gamma)=\mathcal{B}\left(V^{*}, \gamma\right)$. When $\gamma=\operatorname{Id}_{V}$, we write $\mathcal{B}(M)=\mathcal{B}\left(M, \operatorname{Id}_{V}\right)$.

ExAmple 2.4. Let $d: S \rightarrow S \otimes V^{*}$ be the unique derivation of $S$ modules such that $d X=1 \otimes X$ for every element $X \in V^{*}$. If $\left(X_{1}, \ldots, X_{r}\right)$ is a basis of $V^{*}$, then for $P \in S, d P=\sum_{i=1}^{r} \frac{\partial P}{\partial X_{i}} \otimes X_{i}$. Evidently $d$ commutes with the action of $\mathrm{GL}(V)$. Let $S_{+}$denote the ideal of $S$ comprising the functions vanishing at 0 . Since $\mathcal{N}$ is reductive, there exists an $\mathcal{N}$-stable graded subspace $Y$ of $S^{G}$ such that $S_{+}^{G}=\left(S_{+}^{G}\right)^{2} \oplus Y$. Let $\left(P_{1}, \ldots, P_{r}\right)$ be a homogeneous basis of $Y$. Then it is well-known that the natural map $S(Y) \rightarrow S^{G}$ is an $\mathcal{N}$-equivariant isomorphism of algebras (here $S(Y)$ denotes the symmetric algebra on $Y$ ), i.e. that $S^{G} \cong K\left[P_{1}, \ldots, P_{r}\right]$, and that $\left(d P_{1}, \ldots, d P_{r}\right)$ is an $S^{G}$-basis of $\left(S \otimes V^{*}\right)^{G}$. Denote by $\bar{d}$ the composite map $\bar{d}: Y \stackrel{d}{\rightarrow}\left(S \otimes V^{*}\right)^{G} \stackrel{\eta}{\rightarrow}\left(\mathcal{H} \otimes V^{*}\right)^{G}$ where $\eta: S \otimes V^{*} \rightarrow \mathcal{H} \otimes V^{*}$ is the extension to $S \otimes V^{*}$ of the natural map $S \rightarrow S / I \simeq \mathcal{H}$. Then $\bar{d}$ is an isomorphism of degree -1 of $\mathcal{N}$-modules. For each $\iota \in \mathcal{B}(\gamma)$, let $P_{\iota}=\bar{d}^{-1}(\iota)$. Then $\left\{P_{\iota} \mid \iota \in \mathcal{B}(\gamma)\right\}$ is another basis of $Y$, which consists of $\gamma$-eigenfunctions. The $P_{\iota}$ form a set of homogeneous basic $G$-invariants in $S$, and since $\bar{d}$ has degree -1 ,

$$
\operatorname{deg} P_{\iota}=m_{\iota}+1
$$

where $m_{\iota}$ are the usual exponents of $G$. Further, since $\bar{d}$ respects the action of $\mathcal{N}$, we have

$$
\gamma\left(P_{\iota}\right)=\varepsilon_{\iota} P_{\iota}
$$

Definition 2.7. For $\iota \in \mathcal{B}(\gamma)$, write $d_{\iota}=m_{\iota}+1$. The multiset of degrees of $G$ is $\left\{d_{\iota}=\operatorname{deg} P_{\iota} \mid \iota \in \mathcal{B}(\gamma)\right\}$.

Correspondingly, for $\iota \in \mathcal{B}^{*}(\gamma)$, write $d_{\iota}^{*}=m_{\iota}-1$. In this case the $m_{\iota}$ are called the coexponents of $G$, and the multiset of codegrees of $G$ is $\left\{d_{\iota}^{*} \mid \iota \in \mathcal{B}^{*}(\gamma)\right\}$. 
We shall be making use of the following result of Gutkin. In discussing it, we take $\gamma=1$ above, i.e. the theorem will be stated in the "untwisted" context. For any $G$-module $M$ of finite dimension $m$, let $N(M)=\sum_{\iota \in \mathcal{B}(M)} m_{\iota}$. Given $H \in \mathcal{A}$, denote by $G_{H}$ the cyclic (reflection) subgroup of $G$ comprising the elements which fix $H$ pointwise, and set $N_{H}(M)=N\left(\operatorname{Res}_{G_{H}}^{G} M\right)$. If $\xi_{H}$ is the unique non-trivial component character of the representation of $G_{H}$ on $V$, then any character $\xi$ of $G_{H}$ is uniquely expressible as $\xi=\xi_{H}^{e}$, where $0 \leq e \leq e_{H}-1$. Accordingly, if we write $\operatorname{Res}_{G_{H}}^{G} M^{*}=\bigoplus_{i=1}^{m} \xi_{H}^{e_{i}}$ with $0 \leq e_{i} \leq e_{H}-1$, then clearly $N_{H}(M)=\sum_{i=1}^{m} e_{i}$. Observe that for any $G$-module $M, S \otimes \Lambda M^{*}$ is a bigraded associative algebra, where $\operatorname{deg}\left(F \otimes x_{1} \wedge \cdots \wedge x_{j}\right)=(i, j)$ for $F \in S$ homogeneous of degree $i$ and $x_{1}, \ldots, x_{j} \in M^{*}$. The following theorem is due to Gutkin (cf. [OS, 2.10]):

TheOrem 2.8. (Gutkin) Let $y_{1}, \ldots, y_{m}$ be a basis of $M^{*}$. Then the product $\prod_{\iota \in \mathcal{B}(M)} \iota$ in $S \otimes \Lambda M^{*}$ lies in $\left(S \otimes \Lambda^{m} M^{*}\right)^{G}$ and satisfies

$$
\prod_{\iota \in \mathcal{B}(M)} \iota \doteq \prod_{H \in \mathcal{A}} L_{H}^{N_{H}(M)} \otimes y_{1} \wedge y_{2} \wedge \cdots \wedge y_{m}
$$

where $\doteq$ denotes equality up to multiplication by some $\lambda \in K^{\times}$. In particular by comparing degrees, we have $N(M)=\sum_{H \in \mathcal{A}} N_{H}(M)$.

The polynomial $\prod_{H \in \mathcal{A}} L_{H}^{N_{H}(M)}$ will be denoted by $\Psi_{M}$.

Example 2.9. If $H \in \mathcal{A}$, let $e_{H}=\left|G_{H}\right|$. Then since $N_{H}(V)=e_{H}-1$ and $N_{H}\left(V^{*}\right)=1$, we get

$$
\Psi_{V}=\prod_{H \in \mathcal{A}} L_{H}^{e_{H}-1} \quad \text { and } \quad \Psi_{V^{*}}=\prod_{H \in \mathcal{A}} L_{H} .
$$

We shall also require the next result, which is due to Orlik and Solomon (cf. [OS, 3.1]).

Theorem 2.10. (Orlik and Solomon) Let $M$ be a $G$-module of dimension $m$. If $N\left(\Lambda^{m}(M)\right)=N(M)$, then

$$
\left(S \otimes \Lambda\left(M^{*}\right)\right)^{G} \simeq S^{G} \otimes \Lambda\left(\left(\mathcal{H} \otimes M^{*}\right)^{G}\right) .
$$

Equivalently, in the above notation,

$$
\left(\mathcal{H} \otimes \Lambda M^{*}\right)^{G} \simeq \Lambda\left(\left(\mathcal{H} \otimes M^{*}\right)^{G}\right) .
$$


The next lemma (cf. [OS, pp. 79-82]) shows that Theorem 2.10 can be applied to a certain class of representations of $G$ which include the Galois conjugates of $V$.

Lemma 2.11. Suppose $M$ is any $G$-module in which the reflections of $G$ act as reflections. Then $N\left(\Lambda^{m} M\right)=N(M)$, where $m=\operatorname{dim} M$.

Since the Galois conjugates of $V$ clearly satisfy the conditions of (2.11), an immediate consequence is

Corollary 2.12. If $\sigma \in \operatorname{Gal}(K / \mathbb{Q})$, then $N\left(\Lambda^{r}\left(V^{\sigma}\right)\right)=N\left(V^{\sigma}\right)$.

For the convenience of the reader, and also since our proofs may be slightly more straightforward than those in the literature, we provide proofs of Theorem 2.8 and Lemma 2.11 in Appendix 2 below.

\section{Some bilinear forms}

We complete this section by defining some bilinear forms which will be used extensively below. If $\Gamma$ is a subgroup of $\mathcal{N}$ and $M$ is a $\langle G, \Gamma\rangle$-module, the $S$-bilinear form $(S \otimes M) \times\left(S \otimes M^{*}\right) \rightarrow S$, given by $\left(f \otimes x, f^{\prime} \otimes \varphi\right) \mapsto$ $\varphi(x) f f^{\prime}$ is $\langle G, \Gamma\rangle$-equivariant. Therefore it induces by restriction an $S^{G_{-}}$ bilinear form $\langle,\rangle_{M}:(S \otimes M)^{G} \times\left(S \otimes M^{*}\right)^{G} \rightarrow S^{G}$ which is $\Gamma$-equivariant. Take an element $\gamma \in \Gamma$. For $\iota \in \mathcal{B}(M, \gamma)$ and $\jmath \in \mathcal{B}\left(M^{*}, \gamma\right)$, we set

$$
\mathcal{M}_{\iota \jmath}^{M}=\langle\iota, \jmath\rangle_{M} .
$$

Evidently the matrix $\mathcal{M}^{M}=\left(\mathcal{M}_{\iota \jmath}^{M}\right)_{(\iota, \jmath) \in \mathcal{B}(M, \gamma) \times \mathcal{B}\left(M^{*}, \gamma\right)}$ has entries in $S^{G}$, and we have

$$
\gamma\left(\mathcal{M}_{\iota \jmath}^{M}\right)=\varepsilon_{\iota} \varepsilon_{\jmath} \mathcal{M}_{\iota \jmath}^{M} .
$$

Let $\Delta_{M} \in S^{G}$ denote the determinant of $\mathcal{M}^{M}$.

LEMMA 2.14. We have

$$
\Delta_{M}=\Delta_{M^{*}} \doteq \Psi_{M} \Psi_{M^{*}} .
$$

Proof. Let $\left(v_{1}, \ldots, v_{m}\right)$ be a basis of $M$ and $\left(v_{1}^{*}, \ldots, v_{m}^{*}\right)$ the dual basis of $M^{*}$. For $\iota \in \mathcal{B}(M)$ write $\iota=\sum_{k=1}^{m} q_{\iota k}^{*} \otimes v_{k}^{*}$ and for $\jmath \in \mathcal{B}\left(M^{*}\right)$ write $\jmath=\sum_{k=1}^{m} q_{\jmath k} \otimes v_{k}$ where $q_{\jmath k}, q_{\iota k}^{*} \in \mathcal{H} \subset S$. Let $Q=\left(q_{\jmath k}\right)_{\jmath \in \mathcal{B}\left(M^{*}\right), 1 \leq k \leq m}$ and $Q^{*}=\left(q_{\iota k}^{*}\right)_{\iota \in \mathcal{B}(M), 1 \leq k \leq m}$. Then $\Psi_{M} \doteq \operatorname{det} Q^{*}$ and $\Psi_{M^{*}} \doteq \operatorname{det} Q$. Therefore, $\Psi_{M} \Psi_{M^{*}} \doteq(\operatorname{det} Q)\left(\operatorname{det} Q^{*}\right)$. Now $\langle\iota, \jmath\rangle_{M}=\sum_{k=1}^{m} q_{\jmath k} q_{\iota k}^{*}:=r_{\jmath \iota}$, where $Q^{t} Q^{*}=\left(r_{\jmath \iota}\right)_{\jmath \in \mathcal{B}\left(M^{*}\right), \iota \in \mathcal{B}(M)}$. Therefore $\Delta_{M} \doteq \operatorname{det}\left(Q^{t} Q^{*}\right)$, as stated. 
ExAmple 2.16. Write $\mathcal{M}=\mathcal{M}^{V}$ and $\Delta=\Delta_{V}$. Then $\mathcal{M}$ is called the discriminant matrix of $G$ and its determinant $\Delta$ is the discriminant of $G$. From the above, we see

$$
\Delta \doteq \prod_{H \in \mathcal{A}} L_{H}^{e_{H}}
$$

(see (2.15) and Example 2.9).

\section{§3. A twisted polynomial identity}

We start with the following "twisted" version of a result of Orlik and Solomon (cf. [OS, 3.3]).

TheOrem 3.1. Let $M$ be a $\langle G, \gamma\rangle$-module of dimension $m$ such that $N\left(\Lambda^{m}(M)\right)=N(M)$. Then

$$
|G|^{-1} \sum_{g \in G} \frac{\operatorname{det}\left(1-y g \gamma \mid M^{*}\right)}{\operatorname{det}\left(1-x g \gamma \mid V^{*}\right)}=\frac{\prod_{\iota \in \mathcal{B}(M, \gamma)}\left(1-y \varepsilon_{\iota} x^{m_{\iota}}\right)}{\prod_{\iota \in \mathcal{B}(\gamma)}\left(1-\varepsilon_{\iota} x^{d_{\iota}}\right)} .
$$

Proof. We have seen that $S \otimes \Lambda M^{*}$ is a bigraded $K$-vector space. Thus we may define the bi-graded trace $\operatorname{Tr}(\alpha ; x, y) \in K[[x, y]]$ of a bi-graded endomorphism $\alpha$ by

$$
\operatorname{Tr}_{\left(S \otimes \Lambda M^{*}\right)^{G}}(\alpha ; x, y)=\sum_{i, j \geq 0}^{\infty} \operatorname{Tr}\left(\alpha,\left(S \otimes \Lambda M^{*}\right)_{i, j}^{G}\right) x^{i} y^{j} .
$$

We now compute $\operatorname{Tr}_{\left(S \otimes \Lambda M^{*}\right)^{G}}(\gamma ; x, y)$ in two different ways using $(2.10)$. On the left side we use a variant of Molien's formula, while on the right, we use well-known methods for computing graded traces in tensor and exterior algebras (cf. e.g., $[\mathrm{LM}]$ ).

For $\zeta \in K^{\times}$and $g \in \mathrm{GL}(V)$ denote by $V(g, \zeta)$ the $\zeta$-eigenspace of $g$. Clearly $V(g, \zeta)$ coincides with the subspace $V^{\zeta^{-1} g}$ of points of $V$ fixed by $\zeta^{-1} g$.

For any finite dimensional $\langle G, \gamma\rangle$-module $M$, write $U(M, \gamma)$ for the set $\left\{\iota \in \mathcal{B}(M, \gamma) \mid \varepsilon_{\iota}=1\right\}$ and $U_{\#}(M, \gamma)=\mathcal{B}(M, \gamma) \backslash U(M, \gamma)$. Then $U(M, \gamma)$ is a homogeneous basis of $\left(\mathcal{H} \otimes M^{*}\right)^{\langle G, \gamma\rangle}$. In particular, $|U(M, \gamma)|=$ $\operatorname{dim}\left(\mathcal{H} \otimes M^{*}\right)^{\langle G, \gamma\rangle}$.

Since $G$ is finite, as a $G$-module, $V^{*}$ is a Galois conjugate of $V$. However this is not the case for $V^{*}$ regarded as a GL $(V)$-module. Since we include 
elements $\gamma$ in our discussion, the inverses of whose eigenvalues may not be Galois conjugate (setwise) to those of $\gamma$, we need to distinguish between the Galois conjugates of $V$ and those of $V^{*}$. For $\sigma \in \operatorname{Gal}(K, \mathbb{Q})$, write $\mathcal{B}(\sigma, \gamma), U(\sigma, \gamma)$ and $U_{\#}(\sigma, \gamma)$ for $\mathcal{B}\left(V^{\sigma}, \gamma\right), U\left(V^{\sigma}, \gamma\right)$ and $U_{\#}\left(V^{\sigma}, \gamma\right)$ respectively. Similarly, write $\mathcal{B}^{*}(\sigma, \gamma), U^{*}(\sigma, \gamma)$ and $U_{\#}^{*}(\sigma, \gamma)$ for $\mathcal{B}\left(\left(V^{*}\right)^{\sigma}, \gamma\right)$, $U\left(\left(V^{*}\right)^{\sigma}, \gamma\right)$ and $U_{\#}\left(\left(V^{*}\right)^{\sigma}, \gamma\right)$ respectively. Finally, write $U(\gamma), U_{\#}(\gamma)$, $U^{*}(\gamma)$ and $U_{\#}^{*}(\gamma)$ for $U(V, \gamma), U_{\#}(V, \gamma), U\left(V^{*}, \gamma\right)$ and $U_{\#}\left(V^{*}, \gamma\right)$. Thus, for example, $U^{*}(\gamma)=U\left(V^{*}, \gamma\right)$, which is a basis of $(\mathcal{H} \otimes V)^{\langle G, \gamma\rangle}$, and $U(\gamma)=U(V, \gamma)$, which is a basis of $\left(\mathcal{H} \otimes V^{*}\right)^{\langle G, \gamma\rangle}$.

Proposition 3.2. For any $\gamma \in \mathcal{N}$ and any $\sigma \in \operatorname{Gal}(K / \mathbb{Q})$, we have:

(i) If $V \neq V^{G}$, then $\left|U^{*}(\gamma)\right| \geq 1$.

(ii) $|U(\gamma)| \leq|U(\sigma, \gamma)|$ and $|U(\gamma)| \leq\left|U^{*}(\sigma, \gamma)\right|$.

Proof. (i) If $\left(v_{1}, \ldots, v_{r}\right)$ and $\left(X_{1}, \ldots, X_{r}\right)$ are dual bases of $V$ and $V^{*}$ respectively, the element $\sum_{i} X_{i} \otimes v_{i} \in S \otimes V$ is invariant under the whole of $\operatorname{GL}(V)$, and hence a fortiori under $\langle G, \gamma\rangle$. Moreover if $V^{G}=0$, this element lies in $\mathcal{H} \otimes V$, and so $\operatorname{dim}(\mathcal{H} \otimes V)^{\langle G, \gamma\rangle} \geq 1$. More generally, whenever $V \neq V^{G}$, it represents a non-zero invariant element of degree 1 of $S_{G} \otimes V$, whence the statement.

(ii) follows from the same argument as in [LM, Proof of Theorem 2.3], applied to Theorem 3.1 taking $M=V^{\sigma}$ and $M=\left(V^{*}\right)^{\sigma}$ respectively. Note that both these choices of $M$ satisfy the condition of (3.1) by Lemma 2.11.

The following result is deduced from Theorem 3.1 as in [LM, Theorem 2.3]. Note that (3.4) is obtained by applying Theorem 3.1 with $M=V^{\sigma}$ while (3.5) is obtained by applying Theorem 3.1 with $M=\left(V^{*}\right)^{\sigma}$. Theorem 3.1 applies to both cases by Lemma 2.11 .

THEOREm 3.3. If $h: V \rightarrow V$ is a linear transformation, denote by $\operatorname{det}^{\prime}(h)$ the product of the non-zero eigenvalues of $h$. Then we have the following polynomial identities in $K[T]$ for any $\sigma \in \operatorname{Gal}(K / \mathbb{Q})$. In the 
formulae below det always refers to the determinant on $V$.

$$
\sum_{g \in G} T^{\operatorname{dim} V^{g \gamma}} \operatorname{det}^{\prime}(1-g \gamma)^{\sigma-1}=
$$

$$
\begin{cases}0 & \text { if }|U(\gamma)| \neq|U(\sigma, \gamma)|, \\ \prod_{\iota \in U(\sigma, \gamma)}\left(T+m_{\iota}\right) \prod_{\iota \in U_{\#}(\sigma, \gamma)}\left(1-\varepsilon_{\iota}^{-1}\right) \prod_{\iota \in U_{\#}(\gamma)} \frac{d_{\iota}}{1-\varepsilon_{\iota}^{-1}} \quad \text { otherwise. }\end{cases}
$$

$$
(-1)^{r} \sum_{g \in G}(-T)^{\operatorname{dim} V^{g \gamma}} \operatorname{det}^{\prime}(1-g \gamma)^{\sigma-1} \operatorname{det}(g \gamma)^{-\sigma}=
$$

$$
\begin{cases}0 & \text { if }|U(\gamma)| \neq\left|U^{*}(\sigma, \gamma)\right|, \\ \prod_{\iota \in U^{*}(\sigma, \gamma)}\left(T+m_{\iota}\right) \prod_{\iota \in U_{\#}^{*}(\sigma, \gamma)}\left(1-\varepsilon_{\iota}^{-1}\right) \prod_{\iota \in U_{\#}(\gamma)} \frac{d_{\iota}}{1-\varepsilon_{\iota}^{-1}} \quad \text { otherwise. }\end{cases}
$$

We record two special cases of this theorem. They are obtained by taking $\sigma=\operatorname{Id}_{K}$ in (3.4) and (3.5) respectively. Note that (3.7) shall be reinterpreted in (5.8) below.

$$
\sum_{g \in G} T^{\operatorname{dim} V^{g \gamma}}=\prod_{\iota \in U(\gamma)}\left(T+d_{\iota}-1\right) \prod_{\iota \in U_{\#}(\gamma)} d_{\iota}
$$

$$
(-1)^{r} \sum_{g \in G} \operatorname{det}(g \gamma)^{-1}(-T)^{\operatorname{dim} V^{g \gamma}}=
$$

$$
\begin{cases}0 & \text { if }|U(\gamma)| \neq\left|U^{*}(\gamma)\right|, \\ \prod_{\iota \in U^{*}(\gamma)}\left(T+d_{\iota}^{*}+1\right) \prod_{\iota \in U_{\#}^{*}(\gamma)}\left(1-\varepsilon_{\iota}^{-1}\right) \prod_{\iota \in U_{\#}(\gamma)} \frac{d_{\iota}}{1-\varepsilon_{\iota}^{-1}} & \text { otherwise. }\end{cases}
$$

We refer to the elements of $V-\bigcup_{H \in \mathcal{A}} H$ as $\left(G\right.$-)regular, and call $\zeta \in \mathbb{C}^{\times}$ regular for the coset $G \gamma$ if there is an element of $G \gamma$ which has a regular eigenvector with corresponding eigenvalue $\zeta$. In complete analogy with $[\mathrm{LM}]$, we deduce the next statement from (3.7).

Proposition 3.8. The eigenvalue $1 \in K^{\times}$is regular for $G \gamma$ if and only if $|U(\gamma)|=\left|U^{*}(\gamma)\right|$, or equivalently $\operatorname{dim}\left(\mathcal{H} \otimes V^{*}\right)^{\langle G, \gamma\rangle}=\operatorname{dim}(\mathcal{H} \otimes$




Remark 3.9. The element $\gamma \in \mathcal{N}$ may be replaced by $\zeta^{-1} \gamma$, where $\zeta$ is any element of $K^{\times}$, and the formulae of Theorem 3.3 are then correspondingly modified, in a way we shall now describe. Recall that as pointed out above, $V^{\zeta^{-1} g \gamma}=V(g \gamma, \zeta)$. Further, it follows from Remark 2.3 that for any element $\sigma \in \operatorname{Gal}(K / \mathbb{Q}), \varepsilon_{\iota}\left(\zeta^{-1} \gamma\right)=\zeta^{m_{\iota}+\sigma} \varepsilon_{\iota}(\gamma)$ for each basis element $\iota \in \mathcal{B}(\sigma, \gamma)$. Similarly, $\varepsilon_{\iota}\left(\zeta^{-1} \gamma\right)=\zeta^{m_{\iota}-\sigma} \varepsilon_{\iota}(\gamma)$ for every $\iota \in \mathcal{B}^{*}(\sigma, \gamma)$. Therefore, from Definition 2.7, we have $\varepsilon_{\iota}\left(\zeta^{-1} \gamma\right)=\varepsilon_{\iota}(\gamma) \zeta^{d_{\iota}}$ for every $\iota \in \mathcal{B}(\gamma)$ and we have $\varepsilon_{\iota}\left(\zeta^{-1} \gamma\right)=\varepsilon_{\iota}(\gamma) \zeta^{d_{\iota}^{*}}$ for every $\iota \in \mathcal{B}^{*}(\gamma)$.

Remark 3.9 immediately yields the following general form of the criterion (3.8) for regularity, which is the twisted generalisation of the one given in $[\mathrm{LS} 2],[\mathrm{LM}]$.

Corollary 3.10. The element $\zeta \in K^{\times}$is regular for $G \gamma$ if and only if $\left|\left\{\iota \in \mathcal{B}(\gamma) \mid \varepsilon_{\iota} \zeta^{d_{\iota}}=1\right\}\right|=\left|\left\{\jmath \in \mathcal{B}^{*}(\gamma) \mid \varepsilon_{\jmath} \zeta^{d_{\jmath}^{*}}=1\right\}\right|$.

\section{$\S 4$. Parabolic subgroups}

Let $v$ be any point in $V$ and let $C_{G}(v)=\{g \in G \mid g(v)=v\}$; this is a parabolic subgroup of $G$, and contains as a normal subgroup the group $G_{v}$ defined as the subgroup of $C_{G}(v)$ which is generated by reflections which fix $v$. Of course by Steinberg's Theorem (cf. e.g. [L]), the groups $G_{v}$ and $C_{G}(v)$ coincide, but we shall not assume this for the moment, since as a special case of the results of this section we recover the proof of Steinberg's theorem, given in op. cit.

Now $G_{v}$ is a reflection group and $\mathcal{A}\left(G_{v}\right)=\{H \in \mathcal{A}(G) \mid v \in H\}$. Let $\mathcal{N}_{\langle v\rangle}=\{g \in \mathcal{N} \mid g(v) \in K v\}$. Then $\mathcal{N}_{\langle v\rangle}$ contains the reflection group $G_{v}$ as a normal subgroup.

Let $I_{v}$ be the ideal of $S$ generated by the homogeneous elements of positive degree of $S^{G_{v}}$ and write $\mathcal{H}_{v}$ for the space of $G_{v}$-harmonic polynomials, i.e. polynomials which are annihilated by all $G_{v}$-invariant polynomial differential operators with no constant term. Evidently $\mathcal{H}_{v} \subseteq \mathcal{H}$, and $\mathcal{N}_{\langle v\rangle}$ stabilises the decomposition $S=I_{v} \oplus \mathcal{H}_{v}$; further, the natural map $S^{G_{v}} \otimes \mathcal{H}_{v} \rightarrow S$ is an isomorphism of $\mathcal{N}_{\langle v\rangle}$-modules. Notice that if $v$ is $G$-regular, $G_{v}=\{1\}$, and $\mathcal{H}_{v}=K$.

Notation 4.1. Let $N$ be any group and $M=\bigoplus_{i \in \mathbb{Z}} M_{i}$ a $\mathbb{Z}$-graded $K[N]$-module. For any linear character $\theta: N \rightarrow K^{\times}$define $M \stackrel{\text { gr }}{\otimes} \theta$ to be the graded $N$-module $\bigoplus_{i} M_{i} \otimes \theta^{i}$. 
Consider the linear map $\eta_{v}: S \rightarrow \mathcal{H}_{v}$ given by $f \otimes h \in S \simeq S^{G_{v}} \otimes \mathcal{H}_{v} \mapsto$ $f(v) h$. We also denote by $\eta_{v}: \mathcal{H} \rightarrow \mathcal{H}_{v}$ its restriction to $\mathcal{H}$. Then $\eta_{v}$ clearly respects the action of $G_{v}$ on both sides; we investigate how the action of $\mathcal{N}_{\langle v\rangle}$ is transformed. Let $\theta_{v}: \mathcal{N}_{\langle v\rangle} \rightarrow K^{\times}$be the linear character defined by $g(v)=\theta_{v}(g) v$ for every $g \in \mathcal{N}_{\langle v\rangle}$.

LEMMA 4.2. With the above notation, $\eta_{v}$ induces an epimorphism of $\mathcal{N}_{\langle v\rangle}$-modules from $\mathcal{H} \stackrel{\text { gr }}{\otimes} \theta_{v}$ to $\mathcal{H}_{v} \stackrel{\text { gr }}{\otimes} \theta_{v}$.

Proof. Clearly $\eta_{v}$ is linear, and since $\mathcal{H} \supseteq \mathcal{H}_{v}$, it is also evident that $\eta_{v}: \mathcal{H} \rightarrow \mathcal{H}_{v}$ is an epimorphism. It therefore remains only to show that $\eta_{v}$ respects the indicated actions of $\mathcal{N}_{\langle v\rangle}$ on the two spaces.

For $n \in \mathcal{N}_{\langle v\rangle}$, we denote simply by $n$ its action on $S$, and by $\rho(n)$ (resp. $\left.\rho_{v}(n)\right)$ its action on $\mathcal{H} \stackrel{\text { gr }}{\otimes} \theta_{v}\left(\operatorname{resp} . \mathcal{H}_{v} \stackrel{\text { gr }}{\otimes} \theta_{v}\right)$. Then for any element $F=f \otimes h \in\left(S^{G_{v}} \otimes \mathcal{H}_{v}\right) \cap \mathcal{H}$, with $f$ and $h$ homogeneous, we have

$$
\begin{aligned}
\eta_{v}(\rho(n)(f \otimes h)) & =\eta_{v}\left(\theta_{v}(n)^{\operatorname{deg} f+\operatorname{deg} h} n(f) \otimes n(h)\right) \\
& =\theta_{v}(n)^{\operatorname{deg} f+\operatorname{deg} h} n(f)(v) n(h) \\
& =\theta_{v}(n)^{\operatorname{deg} f+\operatorname{deg} h} f\left(n^{-1}(v)\right) n(h) \\
& =\theta_{v}(n)^{\operatorname{deg} f+\operatorname{deg} h} f\left(\theta_{v}(n)^{-1} v\right) n(h) \\
& =\theta_{v}(n)^{\operatorname{deg} h} f(v) n(h) \\
& =\rho_{v}(n)(f(v) h) \\
& =\rho_{v}(n)\left(\eta_{v}(f \otimes h)\right) .
\end{aligned}
$$

Now let $\Gamma$ be any subgroup of $\mathcal{N}_{\langle v\rangle}$ and let $M$ be a finite dimensional $\langle G, \Gamma\rangle$-module. Consider $\mathcal{H} \otimes M$ as a graded module by having regard only to the degree in $\mathcal{H}$ of its elements. Then the $\Gamma$-modules $\left(\mathcal{H} \stackrel{\text { gr }}{\otimes} \theta_{v}\right) \otimes M=$ $(\mathcal{H} \otimes M) \stackrel{\text { gr }}{\otimes} \theta_{v}$ are canonically isomorphic. Observe that by Lemma 4.2 , the $\operatorname{map} \eta_{v}^{M}:=\eta_{v} \otimes \mathrm{Id}:\left(\mathcal{H} \stackrel{\text { gr }}{\otimes} \theta_{v}\right) \otimes M \rightarrow\left(\mathcal{H}_{v} \stackrel{\text { gr }}{\otimes} \theta_{v}\right) \otimes M$ respects the action of $\Gamma$ on both sides. Moreover $\eta_{v}^{M}$ clearly maps the $\Gamma$-submodule $(\mathcal{H} \otimes M)^{G \stackrel{\text { gr }}{\otimes}} \theta_{v}$ $\operatorname{into}\left(\mathcal{H}_{v} \otimes M\right)^{G_{v}} \stackrel{\text { gr }}{\otimes} \theta_{v}$.

TheOREm 4.3. Let $\Gamma$ be any subgroup of $\mathcal{N}_{\langle v\rangle}$ and let $M$ be a finite dimensional $\langle G, \Gamma\rangle$-module. Then the map $\eta_{v}^{M}$ introduced above induces an




Proof. Since $\eta_{v}^{M}$ is $\Gamma$-equivariant by (4.2), it suffices to check that it is an isomorphism of vector spaces. Let $m=\operatorname{dim} M$ and let $y_{1}, \ldots, y_{m}$ be a basis of $M$. Let $u_{1}, \ldots, u_{m}$ (resp. $u_{1}^{v}, \ldots, u_{m}^{v}$ ) be homogeneous bases of $(\mathcal{H} \otimes M)^{G}$ (resp. $\left.\left(\mathcal{H}_{v} \otimes M\right)^{G_{v}}\right)$. Since $(\mathcal{H} \otimes M)^{G} \subset\left(S^{G_{v}} \otimes \mathcal{H}_{v} \otimes M\right)^{G_{v}}=$ $S^{G_{v}} \otimes\left(\mathcal{H}_{v} \otimes M\right)^{G_{v}}$, we may write $u_{i}=\sum_{j} q_{j i} u_{j}^{v}$ for some $q_{j i} \in S^{G_{v}}$.

We now apply Gutkin's Theorem (see (2.8)) in turn to $G$ and $G_{v}$; since $u_{1} \cdots u_{m}=\operatorname{det}\left(q_{i j}\right)_{i, j} u_{1}^{v} \cdots u_{m}^{v}$ we obtain

$$
\operatorname{det}\left(q_{i j}\right)_{i, j}=\prod_{H \in \mathcal{A}(G)-\mathcal{A}\left(G_{v}\right)} L_{H}^{N_{H}(M)} .
$$

But for every $H \in \mathcal{A}(G)-\mathcal{A}\left(G_{v}\right), L_{H}(v) \neq 0$. Hence $\operatorname{det}\left(q_{i j}\right)_{i, j}(v) \neq 0$.

Finally, recall that $\eta_{v}^{M}\left(u_{i}\right)=\sum_{j=1}^{m} q_{j i}(v) u_{j}^{v}$ by definition, whence $\eta_{v}^{M}$ is invertible.

We may apply this result using a similar argument to that given in $[\mathrm{L}]$, to relate the $M$-factors of $G$ and its parabolic subgroups. Let $\zeta \in K^{\times}$and let $v \in V$ be a $\zeta$-eigenvector of $\gamma$, so that $\gamma(v)=\zeta v$. Let $M$ be a $\langle G, \gamma\rangle$ module. Let $G_{v}$ be the stabiliser of $v$ in $G$; since $\gamma \in N_{\mathrm{GL}(V)}\left(G_{v}\right), M$ is also a $\left\langle G_{v}, \gamma\right\rangle$-module, and we may consider the basis $\mathcal{B}^{v}(M, \gamma)$ of $\left(\mathcal{H}_{v} \otimes M^{*}\right)^{G_{v}}$ consisting of homogeneous $\gamma$-eigenvectors. We also define $U^{v}(M, \gamma)$ and $U_{\#}^{v}(M, \gamma)$ as analogues for the pair $\left(G_{v}, \gamma\right)$ of the sets defined earlier for $(G, \gamma)$.

Corollary 4.4. Let $\gamma \in \mathcal{N}_{\langle v\rangle}$, let $\zeta=\theta_{v}(\gamma)$ and let $M$ be a $\langle G, \gamma\rangle$ module. Then the multisets $\left\{\varepsilon_{\iota} \zeta^{m_{\iota}} \mid \iota \in \mathcal{B}(M, \gamma)\right\}$ and $\left\{\varepsilon_{\iota} \zeta^{m_{\iota}} \mid \iota \in\right.$ $\left.\mathcal{B}^{v}(M, \gamma)\right\}$ are equal.

Proof. Since $\theta_{v}(\gamma)=\zeta,\left\{\varepsilon_{\iota} \zeta^{m_{\iota}} \mid \iota \in \mathcal{B}(M, \gamma)\right\}$ is the multiset of eigen-

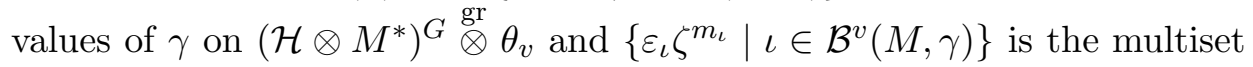
of eigenvalues of $\gamma$ on $\left(\mathcal{H}_{v} \otimes M^{*}\right)^{G_{v}} \stackrel{\text { gr }}{\otimes} \theta_{v}$. Applying Theorem 4.3, with $M$ replaced by $M^{*}$ and $\Gamma$ by $\langle\gamma\rangle$, we obtain that these two multisets are equal.

Corollary 4.5. (Proof of Steinberg's theorem, cf. [L]) In the notation of the first paragraph of this section, we have $C_{G}(v)=G_{v}$.

Proof. Take $\gamma \in C_{G}(v)$. It remains to show $\gamma \in G_{v}$. Since $\gamma v=v$ we take $\zeta=1$ and $M=V$ in (4.4), to obtain, taking into account (2.6), that the multiset $\left\{\varepsilon_{\iota} \mid \iota \in \mathcal{B}^{v}(M, \gamma)\right\}$ consists entirely of 1's, since evidently $\gamma$ acts trivially on $S^{G}$. Hence $\gamma$ acts trivially on $S^{G_{v}}$, whence $\gamma \in G_{v}$. 


\section{The coinvariant algebra as $\langle G, \gamma\rangle$-module}

We shall apply the above considerations to prove a result which generalises that of Stembridge [Ste, 2.3] in two ways. Given a regular number $d$ for $G$, the result in loc. cit. expresses the sum of the graded components of $S_{G}$ (or $\mathcal{H}$ ) of degree congruent to $k$ modulo $d$ as an induced representation. Here we prove an analogous result without the restriction that $d$ be regular; we further extend the statement to the action of $\langle G, \Gamma\rangle$, where $\Gamma$ is any finite subgroup of $\mathcal{N}_{\langle v\rangle}$.

Fix such a subgroup $\Gamma$ of $\mathcal{N}_{\langle v\rangle}$. Note that since $\theta_{v}$ is trivial on $G_{v} \cap \Gamma$, it defines a linear character of $\left\langle G_{v}, \Gamma\right\rangle$ through the isomorphism $\Gamma /\left(G_{v} \cap \Gamma\right) \simeq$ $\left\langle G_{v}, \Gamma\right\rangle / G_{v}$. This will also be referred to as $\theta_{v}$. It thus makes sense to consider the $\left\langle G_{v}, \Gamma\right\rangle$-module $\mathcal{H}_{v} \stackrel{\text { gr }}{\otimes} \theta_{v}$, and more generally for any $k \in \mathbb{Z}$, the $\left\langle G_{v}, \Gamma\right\rangle$-modules $\left(\mathcal{H}_{v} \stackrel{\mathrm{gr}}{\otimes} \theta_{v}\right) \otimes \theta_{v}^{k}$. However, $\theta_{v}^{k}$ defines a character of $\langle G, \Gamma\rangle$ only when $G \cap \Gamma \subset \operatorname{Ker} \theta_{v}^{k}$.

THEOREM 4.6. Let $\mathcal{H}_{i}$ denote the homogeneous component of degree $i$ of the space $\mathcal{H}$ of $G$-harmonic polynomials. Then maintaining the above notation, for any integer $k \in \mathbb{Z}$ there is an isomorphism of $\langle G, \Gamma\rangle$-modules

$$
\bigoplus_{\left\{i \geq 0 \mid G \cap \Gamma \subset \operatorname{Ker} \theta_{v}^{k+i}\right\}} \mathcal{H}_{i} \otimes \theta_{v}^{k+i} \cong \operatorname{Ind}_{\left\langle G_{v}, \Gamma\right\rangle}^{\langle G, \Gamma\rangle}\left(\left(\mathcal{H}_{v} \stackrel{\text { gr }}{\otimes} \theta_{v}\right) \otimes \theta_{v}^{k}\right)
$$

Proof. It suffices to show that both sides have the same inner product with any irreducible $\langle G, \Gamma\rangle$-module $M$. Let $X$ denote the $\langle G, \Gamma\rangle$-module on the left hand-side of the above equation. Then $\langle X, M\rangle_{\langle G, \Gamma\rangle}=\operatorname{dim}((X \otimes$ $\left.\left.M^{*}\right)^{G}\right)^{\Gamma}$. Therefore,

$$
\begin{aligned}
\langle X, M\rangle_{\langle G, \Gamma\rangle} & =\frac{1}{|\Gamma|} \sum_{\gamma \in \Gamma} \operatorname{Tr}\left(\gamma,\left(X \otimes M^{*}\right)^{G}\right) \\
& =\frac{1}{|\Gamma|} \sum_{\gamma \in \Gamma} \sum_{\iota \in \mathcal{B}(M, \gamma)_{k}} \varepsilon_{\iota}(\gamma) \theta_{v}(\gamma)^{m_{\iota}+k},
\end{aligned}
$$

where $\mathcal{B}(M, \gamma)_{k}=\left\{\iota \in \mathcal{B}(M, \gamma) \mid G \cap \Gamma \subset \operatorname{Ker} \theta_{v}^{m_{\iota}+k}\right\}$. Let $[\Gamma /(G \cap \Gamma)]$ be a set of representatives of $\Gamma /(G \cap \Gamma)$. If $\gamma \in[\Gamma /(G \cap \Gamma)]$ and $g \in G \cap \Gamma$, we can take $\mathcal{B}(M, \gamma)=\mathcal{B}(M, \gamma g)$. We then have $\varepsilon_{\iota}(\gamma g)=\varepsilon_{\iota}(\gamma)$ for every 
$\iota \in \mathcal{B}(M, \gamma)$. Therefore

$$
\begin{aligned}
\frac{1}{|\Gamma|} & \sum_{\gamma \in \Gamma} \sum_{\iota \in \mathcal{B}(M, \gamma)} \varepsilon_{\iota}(\gamma) \theta_{v}(\gamma)^{m_{\iota}+k} \\
& =\frac{1}{|\Gamma|} \sum_{\gamma \in[\Gamma /(G \cap \Gamma)]} \sum_{\iota \in \mathcal{B}(M, \gamma)}\left(\varepsilon_{\iota}(\gamma) \theta_{v}(\gamma)^{m_{\iota}+k} \sum_{g \in G \cap \Gamma} \theta_{v}^{m_{\iota}+k}(g)\right) \\
& =\frac{1}{|\Gamma|} \sum_{\gamma \in \Gamma} \sum_{\iota \in \mathcal{B}(M, \gamma)_{k}} \varepsilon_{\iota}(\gamma) \theta_{v}(\gamma)^{m_{\iota}+k} .
\end{aligned}
$$

It follows that

$$
\langle X, M\rangle_{\langle G, \Gamma\rangle}=\frac{1}{|\Gamma|} \sum_{\gamma \in \Gamma}\left(\theta_{v}(\gamma)^{k} \sum_{\iota \in \mathcal{B}(M, \gamma)} \varepsilon_{\iota}(\gamma) \theta_{v}(\gamma)^{m_{\iota}}\right)
$$

Now, let $X^{\prime}$ be the $\langle G, \Gamma\rangle$-module on the right side of (4.7). By Frobenius reciprocity, we have

$$
\left\langle X^{\prime}, M\right\rangle_{\langle G, \Gamma\rangle}=\operatorname{dim}\left(\left(\mathcal{H}_{v} \stackrel{\mathrm{gr}}{\otimes} \theta_{v}\right) \otimes \theta_{v}^{k} \otimes M^{*}\right)^{\langle G, \Gamma\rangle} .
$$

Therefore,

$$
\left\langle X^{\prime}, M\right\rangle_{\langle G, \Gamma\rangle}=\frac{1}{|\Gamma|} \sum_{\gamma \in \Gamma}\left(\theta_{v}(\gamma)^{k} \sum_{\iota \in \mathcal{B}^{v}(M, \gamma)} \varepsilon_{\iota}(\gamma) \theta_{v}(\gamma)^{m_{\iota}}\right) .
$$

The result now follows from (a) and (b), given Corollary 4.4.

Remark 4.8. The special case when $\Gamma=\langle\gamma\rangle$ with $\gamma \in G$ and $v$ is regular is in [Ste, 2.3]. In this case (4.4) essentially amounts to Springer's description of the eigenvalues of a regular element. The general version above could in principle be used to determine the individual graded components of $\mathcal{H}$ as $\langle G, \Gamma\rangle$-modules.

Remark 4.9. Maintain the notation of the previous theorem and assume further that $\gamma \in G$; write $\zeta=\theta_{v}(\gamma)$, and let $d$ be the order of $\zeta$. Then of course $\left\langle G_{v}, \gamma\right\rangle$ is contained in $G$ and Theorem 4.6 can be written as follows.

$$
\bigoplus_{i \equiv-k \bmod d} \mathcal{H}_{i} \cong \operatorname{Ind}_{\left\langle G_{v}, \gamma\right\rangle}^{G}\left(\left(\mathcal{H}_{v} \stackrel{\text { gr }}{\otimes} \theta_{v}\right) \otimes \theta_{v}^{k}\right)
$$


In the special case where $G \simeq \mathfrak{S}_{n}$ is the symmetric group of degree $n, d \leq n$, $\gamma$ is the product of $[n / d]$ disjoint cycles of length $d$ and $G_{v} \simeq \mathfrak{S}_{n-[n / d] d}$, we retrieve a result of Morita and Nakajima [MN].

As a consequence of the previous equation, one obtains (for any $G$ ) that

$$
\operatorname{dim}\left(\bigoplus_{i \equiv-k \bmod d} \mathcal{H}_{i}\right)=\operatorname{dim}\left(\bigoplus_{i \equiv-l \bmod d} \mathcal{H}_{i}\right)
$$

for every $k$ and $l$ in $\mathbb{Z}$ and any natural number $d$ which is the order of an eigenvalue of some element of $G$ (i.e. which divides some degree $d_{i}$ of $G)$. This implies that $\frac{1-t^{d_{i}}}{1-t}$ divides the Poincaré polynomial of $\mathcal{H}$, which of course is well known.

\section{§5. Regularity}

In this section we shall refine and provide a different approach to the regularity result (3.10) above. As usual, we refer to the elements of $V_{\text {reg }}=$ $V-\bigcup_{H \in \mathcal{A}} H$ as $\left(G\right.$-)regular, and call $\zeta \in K^{\times}$regular for the coset $G \gamma$ if there is an element of $G \gamma$ which has a regular eigenvector with corresponding eigenvalue $\zeta$. An element of $G \gamma$ which has a regular eigenvector is called $(G \gamma-)$ regular. Note that $\zeta$ is regular for the coset $G \gamma$ if and only if 1 is regular for the coset $G\left(\zeta^{-1} \gamma\right)$, so that in the context of regularity for cosets, it suffices to consider 1-regularity. In this section we shall give several criteria for the coset $G \gamma$ to contain a regular element.

We start with properties of the quotient variety $V / G$ and the action of $\gamma$ on it. The ring of regular functions on $V / G$ is $K[V / G]=S^{G}$. If $J$ is a subset of $S^{G}$, we denote by $\mathcal{V}(J)$ the closed subvariety of $V / G$ it defines. Let $I(\gamma)$ be the ideal of $S^{G}$ generated by $\left(P_{\iota}\right)_{\iota \in U_{\#}(\gamma)}$ (recall that the $P_{\iota}, \iota \in \mathcal{B}(\gamma)$ form a set of basic homogeneous invariants for $G$, (cf. (2.4)), $\gamma P_{\iota}=\varepsilon_{\iota} P_{\iota}$, and $\left.\iota \in U_{\#}(\gamma) \Longleftrightarrow \varepsilon_{\iota} \neq 1\right)$.

\section{LEMMA 5.1. We have $\mathcal{V}(I(\gamma))=(V / G)^{\gamma}$.}

Proof. Let $K^{\mathcal{B}(\gamma)} \simeq \mathbb{A}^{r}$ be the $K$-vector space of sequences $\left(x_{\iota}\right)_{\iota \in \mathcal{B}(\gamma)}$ of elements of $K$ indexed by $\mathcal{B}(\gamma)$. Then the map $\pi: V \rightarrow K^{\mathcal{B}(\gamma)}, v \mapsto$ $\left(P_{\iota}(v)\right)_{\iota \in \mathcal{B}(\gamma)}$ is a morphism of varieties (corresponding to the inclusion $\left.S^{G} \hookrightarrow S\right)$ which induces an isomorphism $V / G \simeq K^{\mathcal{B}(\gamma)}$. If we endow $K^{\mathcal{B}(\gamma)}$ with the linear action of $\gamma$ given by

$$
\gamma \cdot\left(x_{\iota}\right)_{\iota \in \mathcal{B}(\gamma)}=\left(\varepsilon_{\iota} x_{\iota}\right)_{\iota \in \mathcal{B}(\gamma)},
$$


then, by (2.6), the morphism $\pi$ is $\gamma$-equivariant. But using obvious notation, the space $\left(K^{\mathcal{B}(\gamma)}\right)^{\gamma}$ is naturally identified with $K^{U(\gamma)}$, and the lemma follows.

The variety $V_{\mathrm{reg}} / G$ has a convenient description in these terms. Recall from Example 2.16, that if $\Delta$ is the discriminant polynomial of $G$, we have

$$
V_{\mathrm{reg}} / G=V / G-\mathcal{V}(\Delta)
$$

We next point out twisted generalisations of the results of $[\mathrm{B}]$. The following result has the same proof as $[\mathrm{B}, 1.4,1.6]$; we include it here for the reader's convenience.

Proposition 5.3. Let $G \gamma$ be a reflection coset and let $\zeta \in K^{\times}$. Then

(i) $\zeta$ is regular for $G \gamma$ if and only if $\Delta \notin I\left(\zeta^{-1} \gamma\right)$.

(ii) If $\zeta$ is regular for $G \gamma$ and $U\left(\zeta^{-1} \gamma\right)=\left\{\iota_{0}\right\}$, then $\Delta$ is monic in $P_{\iota_{0}}$.

Proof. (i) Clearly $\zeta$ is regular for $G \gamma$ if and only if 1 is regular for $G \zeta^{-1} \gamma$. Hence we may assume without loss, that $\zeta=1$. But 1 is regular for $G \gamma$ if and only if $\left(V_{\mathrm{reg}} / G\right)^{\gamma}$ is non-empty. By Lemma 5.1 and (5.2), this is the case if and only if $\mathcal{V}(I(\gamma))$ is not contained in $\mathcal{V}(\Delta)$, that is, if and only if $\Delta$ is not in the radical of $I(\gamma)$. The result follows because $I(\gamma)$ is clearly a radical ideal of $S^{G}$.

(ii) Given that $\zeta$ is regular for $G \gamma$ and that $U\left(\zeta^{-1} \gamma\right)=\left\{\iota_{0}\right\}$, it follows from (i) that $\Delta$ is non-zero modulo $I\left(\zeta^{-1} \gamma\right)$, which is generated by $\left\{P_{\iota} \mid\right.$ $\left.\iota \neq \iota_{0}\right\}$. So modulo $I\left(\zeta^{-1} \gamma\right), \Delta \equiv \lambda P_{\iota_{0}}^{k}$ for some $\lambda \in K^{\times}$and some $k \geq 1$. Since $\Delta$ is homogeneous, $\Delta$ is monic in $P_{\iota_{0}}$.

Proposition 5.5 below generalises [LM, Theorem 3.1 (ii)] and is a more precise version of (3.10) above. We shall require some preliminaries before proving it. Let $\mathcal{N}_{v}=\{n \in \mathcal{N} \mid n(v)=v\}$. This is a normal subgroup of $\mathcal{N}_{\langle v\rangle}$ and $\mathcal{N}_{\langle v\rangle} / \mathcal{N}_{v} \simeq K^{\times}$. Let $\Gamma$ be a subgroup of $\mathcal{N}_{v}$. Let $M$ be a $\langle G, \Gamma\rangle$-module. Consider the bilinear form

$$
\begin{aligned}
& \langle,\rangle_{M}^{v}:(\mathcal{H} \otimes M)^{G} \times\left(\mathcal{H} \otimes M^{*}\right)^{G} \longrightarrow \quad K \\
& (f, g) \quad \longmapsto\left(\langle f, g\rangle_{M}\right)(v) .
\end{aligned}
$$

This is simply the evaluation at $v$ of the element $\langle f, g\rangle_{M} \in S^{G}$ (see Section 2). Clearly, $\langle,\rangle_{M}^{v}$ is $\Gamma$-invariant. 
LEMmA 5.4. If $v$ is regular and $\Gamma \subset \mathcal{N}_{v}$, then $\langle,\rangle_{v}^{M}$ is a $\Gamma$-invariant perfect pairing.

Proof. Observe that the discriminant of the bilinear form $\langle,\rangle_{M}^{v}$ is equal to $\Delta_{M}(v)$. But by $(2.15), \Delta_{M}(v) \neq 0$ if $v$ is regular.

Proposition 5.5. Let $\gamma \in \mathcal{N}$. Then the following are equivalent:

(1) 1 is regular for $G \gamma$.

(2) The multisets $\left\{\varepsilon_{\iota}^{-1} \mid \iota \in \mathcal{B}(\gamma)\right\}$ and $\left\{\varepsilon_{\iota} \mid \iota \in \mathcal{B}^{*}(\gamma)\right\}$ are equal.

(3) $|U(\gamma)|=\left|U^{*}(\gamma)\right|$.

Remark 5.6. The equivalence of (1) and (3) follows from an argument similar to [LM, Theorem 3.1 (ii)] (cf. (3.10) above). However the proof we provide here will not make use of the polynomial identities stated in Section 3.

Proof. $\quad(1) \Rightarrow(2)$ Assume that 1 is regular for $G \gamma$. Let $v \in V$ be $G$ regular and such that $g \gamma(v)=v$ for some $g \in G$. Then, by Corollary 5.4, $\left((\mathcal{H} \otimes V)^{G}\right)^{*}$ and $\left(\mathcal{H} \otimes V^{*}\right)^{G}$ are isomorphic $\langle g \gamma\rangle$-modules, via the perfect pairing $\langle,\rangle_{v}$. Therefore, they are isomorphic as $\langle\gamma\rangle$-modules. But $\left\{\varepsilon_{\iota}^{-1} \mid \iota \in\right.$ $\mathcal{B}(\gamma)\}$ is the multiset of eigenvalues of $\gamma$ on $\left((\mathcal{H} \otimes V)^{G}\right)^{*}$ and $\left\{\varepsilon_{\iota} \mid \iota \in \mathcal{B}^{*}(\gamma)\right\}$ is the multiset of eigenvalues of $\gamma$ on $\left(\mathcal{H} \otimes V^{*}\right)^{G}$. The statement follows.

$(2) \Rightarrow(3)$ is trivial.

$(3) \Rightarrow(1)$ Assume that $|U(\gamma)|=\left|U^{*}(\gamma)\right|$. By replacing $\gamma$ by $g_{0} \gamma$ for some $g_{0} \in G$, we may assume that $\operatorname{dim} V^{g \gamma} \leq \operatorname{dim} V^{\gamma}$ for every $g \in G$. We choose $v \in V^{\gamma}$ in "general position", i.e. such that $G_{v}$ acts trivially on $V^{\gamma}$. By Corollary 4.4, the multisets $\left\{\varepsilon_{\iota} \mid \iota \in \mathcal{B}(\gamma)\right\}$ and $\left\{\varepsilon_{\iota} \mid \iota \in \mathcal{B}^{v}(\gamma)\right\}$ are equal, so $|U(\gamma)|=\left|U^{v}(\gamma)\right|$. Similarly, $\left|U^{*}(\gamma)\right|=\left|U^{v *}(\gamma)\right|$. This shows that $\left|U^{v}(\gamma)\right|=\left|U^{v *}(\gamma)\right|$. We now have:

(a) $V^{\gamma} \subset V^{G_{v}}$;

(b) $\operatorname{dim} V^{g \gamma} \leq \operatorname{dim} V^{\gamma}$ for every $g \in G_{v}$;

(c) $\left|U^{v}(\gamma)\right|=\left|U^{v *}(\gamma)\right|$.

We shall show that this implies that $G_{v}=1$. Note that (b) implies that $\operatorname{dim} V^{\gamma}=\left|U^{v}(\gamma)\right|$. Let $V^{\prime}$ be the unique $G_{v^{-}}$stable subspace of $V$ such that $V=V^{G_{v}} \oplus V^{\prime}$. It is $\gamma$-stable, and the homogeneous component of degree 1 
of $\mathcal{H}_{v}$ is $V^{\prime *}$. By (a), $\left(K \otimes V^{\gamma}\right) \oplus\left(V^{*} \otimes V\right)^{\left\langle G_{v}, \gamma\right\rangle}$ is contained in $(\mathcal{H} \otimes V)^{\left\langle G_{v}, \gamma\right\rangle}$. Therefore,

$$
\begin{aligned}
\left|U^{v *}(\gamma)\right| & \geq \operatorname{dim} V^{\gamma}+\operatorname{dim} \operatorname{Hom}_{\left\langle G_{v}, \gamma\right\rangle}\left(V^{\prime}, V\right) \\
& =\left|U^{v}(\gamma)\right|+\operatorname{dim} \operatorname{Hom}_{\left\langle G_{v}, \gamma\right\rangle}\left(V^{\prime}, V\right) .
\end{aligned}
$$

It follows from (c) that $\operatorname{dim} \operatorname{Hom}_{\left\langle G_{v}, \gamma\right\rangle}\left(V^{\prime}, V\right)=0$. Thus $V^{\prime}=0$, and $G_{v}=1$ as required.

Remark 5.7. The right-hand side of (3.7) vanishes unless 1 is regular for $G \gamma$. Hence it may be simplified as follows. All $g \gamma$ which contribute to the highest power of $T$ on the left side of (3.7) are conjugate, and by [Sp, $6.4(\mathrm{v})]$ have the same determinant, which is equal to $\prod_{\iota \in \mathcal{B}(\gamma)} \varepsilon_{\iota}^{-1}$. This in turns yields a formula for $\frac{\prod_{\iota \in U_{\#}^{*}(\zeta)}\left(1-\varepsilon_{\iota}^{-1}\right)}{\prod_{\iota \in U_{\#}(\zeta)}\left(1-\varepsilon_{\iota}^{-1}\right)}$, which may be substituted into (3.7). The result is

$$
\begin{aligned}
& \sum_{g \in G} \operatorname{det}(g \gamma) T^{\operatorname{dim} V^{g \gamma}}= \\
& \begin{cases}0 & \text { if }|U(\gamma)| \neq\left|U^{*}(\gamma)\right|, \\
\prod_{\iota \in \mathcal{B}(\gamma)} \varepsilon_{\iota}^{-1} \prod_{\iota \in U_{\#}^{*}(\gamma)}\left(T-d_{\iota}^{*}-1\right) \prod_{\iota \in U_{\#}(\gamma)} d_{\iota} & \text { otherwise. }\end{cases}
\end{aligned}
$$

Our final observation in this section is that if $\Delta$ is monic in some basic invariant, then there is a natural regular number.

Corollary 5.9. Suppose that the discriminant $\Delta$ is monic in $P_{\iota_{0}}$ for some $\iota_{0} \in \mathcal{B}(\gamma)$. Let $\zeta \in K^{\times}$be such that $\zeta^{d_{\iota_{0}}}=\varepsilon_{\iota_{0}}^{-1}$. Then $\zeta$ is regular for $G \gamma$. In particular, the multisets $\left\{\varepsilon_{\iota} \zeta^{d_{\iota}} \mid \iota \in \mathcal{B}(\gamma)\right\}$ and $\left\{\left(\varepsilon_{\iota} \zeta^{d_{\iota}^{*}}\right)^{-1} \mid \iota \in\right.$ $\left.\mathcal{B}^{*}(\gamma)\right\}$ are equal.

Proof. Note that $\iota_{0} \in U\left(\zeta^{-1} \gamma\right)$ by Remark 2.3. Therefore, by assumption, $\Delta$ does not belong to the ideal $I\left(\zeta^{-1} \gamma\right)$. So, by Proposition 5.3 (i), $\zeta$ is regular for $G \gamma$. Now, the last assertion follows from Proposition 5.5 and from Remark 2.3.

\section{$\S 6 . \quad$ A twisted generalisation of Coxeter elements}

In this section we focus attention on "well-generated" reflection groups. These include the finite Coxeter groups, the Shephard groups, i.e. symmetry groups of regular polytopes, and some others. To define them, we have the 
ObSERvation 6.1. (Orlik and Solomon) Let $G$ be an irreducible reflection group in $V$. Suppose the degrees and codegrees of $G$ are ordered so that $d_{1} \leq d_{2} \leq \cdots \leq d_{r}$ and $d_{1}^{*} \geq d_{2}^{*} \geq \cdots \geq d_{r}^{*}$. Then the following two statements are equivalent.

(i) $G$ is generated by $r=\operatorname{dim} V$ reflections.

(ii) We have $d_{i}+d_{i}^{*}=d_{r}$ for $i=1,2, \ldots, r$.

The only (currently) known proof of (6.1) is empirical. A reflection group satisfying the equivalent conditions of (6.1) is called well-generated.

Henceforth, we shall consistently write $\mathcal{B}(\gamma)=\left(\iota_{1}, \ldots, \iota_{r}\right)$ and $\mathcal{B}^{*}(\gamma)=$ $\left(\iota_{1}^{*}, \ldots, \iota_{r}^{*}\right)$ and we set $d_{\iota_{i}}=d_{i}$ and $d_{\iota_{i}^{*}}^{*}=d_{i}^{*}$. Write $P_{i}=P_{\iota_{i}}$, so that $\operatorname{deg} P_{i}=d_{i}$. We also assume that this numbering satisfies $d_{1} \leq \cdots \leq d_{r}$ and $d_{1}^{*} \geq \cdots \geq d_{r}^{*}$. We also set $\varepsilon_{\iota_{i}}=\varepsilon_{i}$ and $\varepsilon_{\iota_{i}^{*}}=\varepsilon_{i}^{*}$. The next result is part of Bessis' [B2, Theorem 2.2]. Again for convenience, we sketch a proof.

Proposition 6.2. Suppose $G$ is any irreducible reflection group which satisfies $d_{i}+d_{i}^{*} \leq d_{r}$ for all $i$. Then

(i) Any primitive $d_{r}^{\text {th }}$ root of unity is regular for $G$.

(ii) We have $0<d_{i}+d_{j}^{*}<2 d_{r}$ for any pair $(i, j)$.

(iii) Let $I_{0}$ be the ideal of $S^{G}$ generated by $\left\{P_{i} \mid i \neq r\right\}$. Then modulo $I_{0}$, the discriminant matrix $M \equiv P_{r} C$, where $C=\left(c_{i j}\right)_{i, j}$ is a nonsingular matrix with entries in $K$.

(iv) If $d_{i}+d_{j}^{*} \neq d_{r}, c_{i j}=0$.

(v) We have $r d_{r}=N+N^{*}$ and $d_{i}+d_{i}^{*}=d_{r}$ for every $i$.

(vi) Partition $\{1, \ldots, r\}$ into subsets, where $i, j$ are in the same subset if $d_{i}=d_{j}$. Then $C$ is diagonal by block for this decomposition and each block is non-singular.

(vii) $G$ is well-generated.

Proof. Since $G$ is irreducible, we have $d_{i}^{*} \geq 1$ for every $i \leq r-1$. Therefore, by assumption $1 \leq d_{i} \leq d_{r}-1$ and $1 \leq d_{i}^{*} \leq d_{r}-1$ if $i \neq r$. So, if $\zeta_{0}$ is a primitive $d_{r}^{\text {th }}$ root of unity, then $U\left(\zeta_{0}^{-1} \operatorname{Id}_{V}\right)=\left\{\iota_{r}\right\}$ and $U^{*}\left(\zeta_{0}^{-1} \operatorname{Id}_{V}\right)=\left\{\iota_{r}^{*}\right\}$, whence $\zeta_{0}$ is regular for $G$ by the criterion (5.5), proving (i); (ii) is a simple consequence of our assumption on the degrees. By $(5.3), \Delta \equiv c P_{r}^{k} \bmod I\left(\zeta_{0}^{-1} \mathrm{Id}_{V}\right)$, with $c \in K^{\times}$by regularity, and by degree, $k=\left(N(V)+N\left(V^{*}\right)\right) / d_{r}$. Now the entries of $\mathcal{M}$ are homogeneous 
polynomials in $S^{G}$, and by (ii), when written as polynomials in $P_{r}$ with coefficients in $K\left[\left\{P_{i} \mid i \neq r\right\}\right]$, have degree (in $P_{r}$ ) 0 or 1 . The statements (iii), (iv) and the first statement of (v) follow immediately. But, $N(V)+N\left(V^{*}\right)=\sum_{i=1}^{r}\left(d_{i}+d_{i}^{*}\right) \leq r d_{r}=N(V)+N\left(V^{*}\right)$. So $d_{i}+d_{i}^{*}=d_{r}$ for every $i$. This proves (v). (vi) now follows from (iv) and (v) while (vii) follows from (6.1).

The next result refines (5.9) in the case of well-generated groups. It may be regarded as a generalisation of the fact that when $G$ is real and crystallographic, the Coxeter number $h$ (which is the highest degree $d_{r}$ ), and any primitive $h^{\text {th }}$ root of unity is regular, with corresponding regular conjugacy class the Coxeter class of $G$.

Proposition 6.3. Suppose that $G$ is irreducible and well-generated. Let $\zeta \in K^{\times}$. Then:

(i) there is a permutation $\sigma$ of $\{1, \ldots, r\}$ such that for each $i, d_{i}=d_{\sigma(i)}$ and $\varepsilon_{\sigma(i)}^{*}=\varepsilon_{i}^{-1} \varepsilon_{r}$.

(ii) If $\zeta \in K^{\times}$is such that $\zeta^{d_{r}}=\varepsilon_{r}^{-1}$, then $\zeta$ is regular for the coset $G \gamma$.

Proof. By Proposition 6.2 (vi), there is a permutation $\sigma$ of $\{1, \ldots, r\}$ such that $d_{\sigma(i)}=d_{i}$ and $c_{i, \sigma(i)} \neq 0$ for all $i$. Hence $\mathcal{M}_{i, \sigma(i)}=c_{i, \sigma(i)} P_{r}+$ other terms, from which it follows that $\gamma\left(\mathcal{M}_{i, \sigma(i)}\right)=\varepsilon_{r} \mathcal{M}_{i, \sigma(i)}$. But by (2.13), $\gamma\left(\mathcal{M}_{i, \sigma(i)}\right)=\varepsilon_{i} \varepsilon_{\sigma(i)}^{*} \mathcal{M}_{i, \sigma(i)}$.

Since $G$ is well-generated, we have $d_{i}+d_{i}^{*}=d_{r}$. Therefore, by (i), $\varepsilon_{\sigma(i)}^{*} \zeta_{\sigma(i)}^{d^{*}}=\varepsilon_{i}^{-1} \zeta^{-d_{i}}$. In view of the criterion (5.5), (ii) follows from this observation (see also Remark 3.9).

Finally, observe that when $G$ is real (and hence is a finite Coxeter group) we have, using our orderings, $d_{i}^{*}=d_{r+1-i}-2$ and $\varepsilon_{i}^{*}=\varepsilon_{r+1-i}$. Thus, applying (i), we deduce that there is a degree-preserving permutation $\sigma$ such that $\varepsilon_{i} \varepsilon_{r+1-\sigma(i)}=\varepsilon_{r}$.

\section{$\S 7$. Existence of regular elements in cosets}

We shall prove ${ }^{2}$

\footnotetext{
${ }^{2}$ As mentioned in the footnote to the Introduction, Theorem 7.1 also appears, with a different proof, in [Ma].
} 
THEOREM 7.1. There is a semisimple element $z \in \mathrm{GL}(V)$ which centralises $\langle G, \gamma\rangle$ such that the reflection coset $z \gamma G$ has a regular eigenvalue (or element).

As an easy consequence, we have

Corollary 7.2. If $V$ is irreducible as $\langle G, \gamma\rangle$-module, then $G \gamma$ has a regular eigenvalue.

In particular,

Corollary 7.3. If $V$ is irreducible as $G$-module, then $G \gamma$ has a regular eigenvalue.

We begin with a reduction to the case (7.3), which involves arguments similar to those in [BL, Prop. 6.9].

LEMMA 7.4. We have the implications $(7.3) \Rightarrow(7.2) \Rightarrow(7.1)$.

Proof. To see that $(7.2) \Rightarrow(7.1)$, suppose that $V=\bigoplus_{i} V_{i}$ is a decomposition of $V$ into irreducible $\langle G, \gamma\rangle$-submodules. Then correspondingly $G=G_{1} \times G_{2} \times \cdots$, and $\gamma=\bigoplus_{i} \gamma_{i}$, where $G_{i}$ acts as a reflection group in $V_{i}$ and trivially on $V_{j}$ for $j \neq i$, and $\gamma_{i} \in \operatorname{GL}\left(V_{i}\right)$ normalises $G_{i}$. The set $\mathcal{A}$ of reflecting hyperplanes of $G$ is the union of the sets $\mathcal{A}_{i}$ of reflecting hyperplanes of the $G_{i}$. By (7.2) there are elements $g_{i} \in G_{i}$ and $v_{i} \in V_{i}$ such that $\gamma_{i} g_{i} v_{i}=\zeta_{i} v_{i}$, and $v_{i}$ is $G_{i}$-regular in $V_{i}$. Take $z=\bigoplus_{i} \zeta_{i}^{-1} \operatorname{Id}_{V_{i}}$, $g=\left(g_{1}, g_{2}, \ldots\right)$, and $v=\bigoplus_{i} v_{i}$. Then $v$ is $G$-regular and $z \gamma g v=v$, proving (7.1).

Now assume (7.3), and suppose that $V$ is irreducible as $\langle G, \gamma\rangle$-module. Then as in Remark 2.1, $V=V_{1} \oplus \cdots \oplus V_{k}$ and correspondingly $G=G_{1} \times$ $\cdots \times G_{k}$. Then all $\left(G_{i}, V_{i}\right)$ are isomorphic, and are permuted cyclically by $\gamma$. Thus $\gamma^{k}$ fixes all the $V_{i}$, and in particular normalises $G_{1}$ on $V_{1}$, so that by (7.3), there are elements $g_{1} \in G_{1}$ and $v_{1}$ regular in $V_{1}$ such that $\gamma^{k} g_{1} v_{1}=$ $\zeta_{1} v_{1}$. Let $\zeta \in \mathbb{C}$ satisfy $\zeta^{-k}=\zeta_{1}$, let $g=\left(1,1, \ldots, 1, \gamma^{k-1} g_{1} \gamma^{-(k-1)}\right) \in G$, and $v=v_{1} \oplus \zeta \gamma v_{1} \oplus(\zeta \gamma)^{2} v_{1} \oplus \cdots \oplus(\zeta \gamma)^{k-1} v_{1} \in V$. Then $v$ is $G$-regular, and $\gamma g v=\zeta^{-1} v$. Hence $(7.3) \Rightarrow(7.2)$.

It follows from Lemma 7.4, that it suffices to prove (7.3), and hence we take $V$ to be an irreducible $G$-module. The next lemma deals with an obvious case. 
LEMMA 7.5. If $G$ is irreducible and $\gamma$ induces an inner automorphism of $G$, then (7.3) holds.

Proof. By hypothesis, there exists $g \in G$ such that $g \gamma$ is central in $\langle G, \gamma\rangle$ so is scalar. The result is then obvious.

At this point, it would be sufficient to inspect the list of cosets $G \gamma$ such that $G$ is irreducible and $\gamma$ induces a non-inner automorphism of $G$ which is given in [BMM, 3.13]. The table of regular eigenvalues are then given in the table at the end of this paper. However, we will provide some further reductions which cover all the cases to be checked. First, we reduce the proof further to the case of "minimal groups", which are defined as follows.

For any integer $d$, let $\zeta_{d}$ be a primitive $d^{\text {th }}$ root of unity. Then (cf. [LS1], [LS2]) all maximal $\zeta_{d}$ eigenspaces $E$ of elements of $G$ are conjugate under $G$, and the group $G(d):=N_{G}(E) / C_{G}(E)$ is a reflection group in $E$; the subquotient $G(d)$ is unique up to conjugacy in $G$, and is irreducible if $G$ is [LS2]. The regular case is when $C_{G}(E)=1$, in which case $G(d)$ is a subgroup of $G$. Say that $G$ is minimal if $\operatorname{dim} V>1$ and there is no non-trivial subgroup $G(d)<G$ with $d$ regular. Equivalently, if $a(d), b(d)$ respectively denote the number of degrees and codegrees divisible by $d$, then $a(d)=b(d)$ implies that $a(d)=0$ or $r(=\operatorname{dim} V)$. Note that if $\operatorname{dim} V=1$, (7.3) is trivially true.

LEMMA 7.6. Theorem 7.1 is true for irreducible $G$ if it is true for irreducible minimal $G$.

Proof. If $\gamma$ normalises $G$ and $E=V\left(g, \zeta_{d}\right)$ is a maximal $\zeta_{d}$ eigenspace, then $\gamma E=V\left(\gamma g \gamma^{-1}, \zeta_{d}\right)$ is also a maximal $\zeta_{d}$ eigenspace, whence there is an element $x \in G$ such that $\gamma E=x E$, so that $x^{-1} \gamma$ normalises $G(d)$, which is irreducible by [LS2, Theorem A]. If $v \in E$ is a $G(d)$-regular eigenvector for $\gamma y \in x^{-1} \gamma G(d)$, then since the reflecting hyperplanes of $G(d)$ are the intersections with $E$ of those of $G$, and $E$ is not contained in any hyperplane of $G$, it follows that $v$ is $G$-regular. Thus Theorem 7.1 holds for $G$ if it holds for $G(d)$. Repeating this argument, we arrive at a case where $G(d)$ is minimal.

Our final lemma treats a case which arises frequently.

LEMmA 7.7. Assume that $G$ is irreducible and that there exists $\gamma_{0} \in \mathcal{N}$ such that $\langle G, \gamma\rangle \subset\left\langle G, \gamma_{0}\right\rangle$ and $\left\langle G, \gamma_{0}\right\rangle$ is a well-generated finite reflection subgroup of $\mathrm{GL}(V)$. Then $G \gamma$ has a regular eigenvalue. 
Proof. If $G$ is well-generated, the conclusion follows from Proposition 6.3 (ii). Hence we assume that $G$ is not well-generated. By hypothesis, there exists $k \in \mathbb{Z}$ such that $G \gamma_{0}^{k}=G \gamma$. So if $\zeta$ is a regular eigenvalue for $G \gamma_{0}$, then $\zeta^{k}$ is a regular eigenvalue for $G \gamma$. Hence we are reduced to the case $\gamma=\gamma_{0}$. Now let $Y$ be as in Example 2.4. Then $S\left(V^{*}\right)^{\langle G, \gamma\rangle} \simeq S(Y)^{\gamma}$ is a polynomial algebra, so $\gamma$ acts on $Y$ as a reflection. Let $i_{0}$ be the unique element of $\{1,2, \ldots, r\}$ such that $\varepsilon_{i_{0}} \neq 1$ and let $e$ be the order of $\varepsilon_{i_{0}}$. Write $\widetilde{G}=\langle G, \gamma\rangle$. Let $\tilde{d}_{1} \leq \cdots \leq \tilde{d}_{r}$ be the degrees of $\widetilde{G}$ and let $\tilde{d}_{1}^{*} \leq \cdots \leq \tilde{d}_{r}^{*}$ be its codegrees. Since $|U(\gamma)|=r-1$, two cases may occur (see Proposition 3.2 (ii)).

If $\left|U^{*}(\gamma)\right|=r-1$, then $\gamma$ is 1-regular.

If $\left|U^{*}(\gamma)\right|=r$, this means that $\gamma$ acts trivially on $(\mathcal{H} \otimes V)^{G}$. In particular, $\tilde{d}_{i}^{*}=d_{i}^{*}$ for every $i$. Also, $S(Y)^{\gamma} \simeq S\left(V^{*}\right)^{\widetilde{G}}$ is a polynomial algebra generated $P_{1}, \ldots, P_{i_{0}-1}, P_{i_{0}}^{e}, P_{i_{0}+1}, \ldots, P_{r}$. Therefore, since $\widetilde{G}$ is wellgenerated and $G$ is not well-generated, it follows from Proposition 6.2 that $\left(\tilde{d}_{1}, \ldots, \tilde{d}_{r}\right)=\left(d_{1}, \ldots, d_{i_{0}-1}, d_{i_{0}+1}, \ldots, d_{r}, e d_{i_{0}}\right)$. Now, let $\zeta$ be such that $\zeta^{d_{i_{0}}}=\varepsilon_{i_{0}}^{-1}$. Then, since $\tilde{d}_{i}+\tilde{d}_{i}^{*}=e d_{i_{0}}$ and $\zeta^{e d_{i_{0}}}=1, \varepsilon_{i}=1$ if $i \neq i_{0}$, and that $\varepsilon_{i}^{*}=1$ for every $i$ and since we have:

- If $1 \leq i \leq i_{0}-1$, then $d_{i}+d_{i}^{*}=e d_{i_{0}}$ and $\left(\varepsilon_{i} \zeta^{d_{i}}\right)^{-1}=\varepsilon_{i}^{*} \zeta^{d_{i}^{*}}$.

- $\varepsilon_{i_{0}} \zeta^{d_{i_{0}}}=1=\varepsilon_{r} \zeta^{d_{r}^{*}}$.

- If $i_{0}+1 \leq i \leq r$, then $d_{i}+d_{i-1}^{*}=e d_{i_{0}}$ and $\left(\varepsilon_{i} \zeta^{d_{i}}\right)^{-1}=\varepsilon_{i-1}^{*} \zeta^{d_{i-1}^{*}}$.

Therefore, the multisets $\left\{\varepsilon_{i} \zeta^{d_{i}} \mid 1 \leq i \leq r\right\}$ and $\left\{\left(\varepsilon_{i}^{*} \zeta^{d_{i}^{*}}\right)^{-1} \mid 1 \leq i \leq r\right\}$ are equal. So $\zeta$ is a regular eigenvalue for $G \gamma$ by Proposition 5.5.

We are now able to give the

Proof of Theorem 7.1. The list of cosets $G \gamma$ such that $G$ is irreducible and $\gamma$ induces a non-inner automorphism of $G$ is given in [BMM, 3.13]. Among them, the minimal ones are (up to multiplication by a scalar):

1. $G(d e, e, r) \gamma$ when $r \mid e, d>1$ and $e>1$ where $\gamma \in G(d e, 1, r)$.

2. $G(4,2,2) \gamma$ where $\langle G(4,2,2), \gamma\rangle=G_{6}$.

3. $G_{7} \gamma$ where $\left\langle G_{7}, \gamma\right\rangle=G_{15}$.

Since $G(d e, 1, r), G_{6}$ and $G_{15}$ are well-generated, these cases are disposed of by Lemma 7.7. The proof of the theorem is now completed by invoking Lemmas 7.4, 7.5, and 7.6. 


\section{$\S 8$. Reflection quotients of reflection groups}

Let $L$ be a normal subgroup of $G$ and denote by $\bar{G}=G / L$ the corresponding quotient. Let $E^{*}$ be a graded complement of $\left(S_{+}^{L}\right)^{2}$ in $S_{+}^{L}$, so that $S_{+}^{L}=E^{*} \oplus\left(S_{+}^{L}\right)^{2}$. Evidently $E^{*}$ has basis a set of homogeneous generators of the invariant ring $S^{L}$. Let $\mathcal{N}_{L}$ be the normalizer of $L$ in $\operatorname{GL}(V)$. For this section only, we denote the normalizer of $G$ in $\operatorname{GL}(V)$ by $\mathcal{N}_{G}$. Since $\mathcal{N}_{L}$ is a reductive group, we may assume that $E^{*}$ is chosen to be stable under the action of $\mathcal{N}_{L}$. Let $E$ be the (graded) dual of $E^{*}$, and denote by $\bar{S}$ the symmetric algebra of $E^{*}$.

Then $E$ is isomorphic to the tangent space of the variety $V / L$ at 0 . The quotient $\bar{G}$ acts on $E$, and we shall be interested in this section in the case where this is a reflection group action, a situation which has been studied in [BBR]. In that case $\mathcal{N}_{G} \cap \mathcal{N}_{L}$ also acts on $E$, normalising the $\bar{G}$-action, and we shall relate the various twisted invariants of reflection cosets of $G$ and $\bar{G}$.

The algebra homomorphism $\tau: S\left(E^{*}\right) \rightarrow S^{L}$ which extends the inclusion $E^{*} \hookrightarrow S^{L}$ is easily seen to be surjective (see for instance [BBR, Lemma 2.1]) and $\mathcal{N}_{L}$-equivariant. Denote by $I$ its $\left(\mathcal{N}_{L}\right.$-stable) kernel. Then we have a commutative diagram

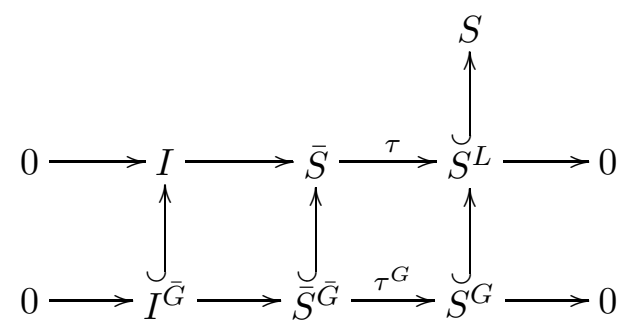

in which the rows are exact.

Note that the surjective morphisms $\tau$ and $\tau^{G}$ induce closed immersions

$$
V / L \hookrightarrow E \quad \text { and } \quad V / G \hookrightarrow E / \bar{G} .
$$

We assume henceforth that $\bar{G}$ acts on $E$ as a reflection group. By [BBR, Theorem 3.2], this is equivalent to requiring that

(1) $V / L$ is a complete intersection variety, and

(2) $I$ is generated by $I^{\bar{G}}$ (as an ideal of $\bar{S}$ ). 
ExAmPLE 8.2. The classification of all such pairs $(G, L)$ is given in [BBR, §4]. If $L$ is generated by reflections, then it is straightforward that $G / L$ is always generated by reflections. We give here another example (for more details, see [BBR, §4.8]). Assume that $G=G_{31}$ and that $L$ is its maximal normal 2-subgroup. Then $L \subset \mathrm{SL}(V),|L|=64,|G / L|=720, E$ is of dimension 5 and concentrated in degree 4 , since $S^{L}$ is generated by 5 polynomials of degree 4 . Here $G / L$ acts faithfully on $E$ as a group generated by reflections, isomorphic to the symmetric group $\mathfrak{S}_{6}$ in its irreducible reflection representation. Note that $G / L$ is well-generated while $G=G_{31}$ is not.

\subsection{Coinvariants}

Denote by $\bar{S}_{\bar{G}}$ the algebra of coinvariants of $\bar{G}$. We shall relate this algebra to the algebra $S_{G}$ of coinvariants of $G$.

Proposition 8.3. The homomorphism $\tau: \bar{S} \rightarrow S^{L}$ introduced above induces an isomorphism of graded algebras

$$
\bar{\tau}: \bar{S}_{\bar{G}} \longrightarrow\left(S_{G}\right)^{L}
$$

which commutes with the action of $\mathcal{N}_{G} \cap \mathcal{N}_{L}(\supseteq G)$.

Proof. The composite of $\tau$ with the inclusion $S^{L} \hookrightarrow S$ maps $\bar{S}$ to $S$, and $\bar{S}_{+}^{\bar{G}}$ to $S_{+}^{G}$. Hence it induces a homomorphism $\bar{\tau}: \bar{S}_{\bar{G}} \rightarrow S_{G}$, whose image is evidently in $\left(S_{G}\right)^{L}$. The equivariance with respect to $\mathcal{N}_{G} \cap \mathcal{N}_{L}$ is clear.

To prove that $\bar{\tau}$ is an isomorphism, first note that $\bar{\tau}$ is surjective since $\tau$ is, because $L$ acts semisimply on $S$. But $\operatorname{dim} \bar{S}_{\bar{G}}=|\bar{G}|=\operatorname{dim}\left(S_{G}\right)^{L}$, whence $\bar{\tau}$ is also injective.

Let $\overline{\mathcal{H}}$ be the space of $\bar{G}$-harmonic polynomial functions on $E$, and as above, $\mathcal{H}$ be the corresponding space for $G$ on $V$.

COROLlary 8.4. The isomorphism $\bar{\tau}$ of (8.3) induces an isomorphism of $\mathcal{N}_{G} \cap \mathcal{N}_{L}$-spaces $: \overline{\mathcal{H}} \rightarrow \mathcal{H}^{L}$, which we shall also denote by $\bar{\tau}$.

Proof. Each coset of the ideal $S . S_{+}^{G}$ of $S$ contains a unique $G$-harmonic polynomial. This provides a canonical $\mathcal{N}_{G^{-}}$equivariant isomorphism of vector spaces : $S_{G} \rightarrow \mathcal{H}$. Similarly we have a canonical $\mathcal{N}_{G} \cap \mathcal{N}_{L^{-}}$equivariant canonical isomorphism $: \overline{\mathcal{H}} \rightarrow S_{\bar{G}}$. If we compose $\bar{\tau}$ with these isomorphisms, taking (8.3) into account, we obtain the desired isomorphism. 


\subsection{Comparison of $M$-factors}

Let $\Gamma$ be a subgroup of $\mathcal{N}_{G} \cap \mathcal{N}_{L}$, and write $\bar{\Gamma} \cong \Gamma /(\Gamma \cap L)$ for its image in $\operatorname{GL}(E)$. Then $\bar{\Gamma}$ normalizes $\bar{G}$ and $\langle G, \Gamma\rangle / L \simeq\langle\bar{G}, \bar{\Gamma}\rangle$. Let $M$ be a $\langle\bar{G}, \bar{\Gamma}\rangle$-module, or equivalently, a $\langle G, \Gamma\rangle$-module on which $L$ acts trivially. Then $\left(S_{G} \otimes M\right)^{G}=\left(\left(S_{G}\right)^{L} \otimes M\right)^{\bar{G}}$. Hence in view of (8.3) we have an isomorphism of $\Gamma$-modules (on which $\Gamma \cap L$ acts trivially)

$$
\left(\bar{S}_{\bar{G}} \otimes M\right)^{\bar{G}} \stackrel{\bar{\tau} \otimes \operatorname{Id}_{M}}{\longrightarrow}\left(S_{G} \otimes M\right)^{G} .
$$

In (8.5), we use the $G$-equivariance of $\bar{\tau}$ to restrict to the $G$-fixed points, noting that on the left, $G$ acts via $\bar{G}$, since $L$ acts trivially. We denote the map of (8.5) by $\tau_{M}$. Similarly, $\tau_{M}$ will also denote the isomorphism of $\Gamma$-modules $(\overline{\mathcal{H}} \otimes M)^{\bar{G}} \rightarrow(\mathcal{H} \otimes M)^{G}$ (cf. Corollary 8.4).

Next assume that the element $\gamma \in \Gamma$ acts semisimply on $M$. Let $\bar{\gamma}$ be its image in $\bar{\Gamma}$. Then $\bar{\gamma}$ is semisimple, and the next statement follows easily from the above remarks.

Lemma 8.6. Let $\mathcal{B}(M, \bar{\gamma})$ be a basis of $(\overline{\mathcal{H}} \otimes M)^{\bar{G}}$ consisting of $\bar{\gamma}$ eigenvectors. Then $\left(\tau_{M}(\iota)\right)_{\iota \in \mathcal{B}(M, \bar{\gamma})}$ is a basis of $(\mathcal{H} \otimes M)^{G}$ consisting of $\gamma$-eigenvectors.

As an immediate consequence, we have

Corollary 8.7. The $M$-factors of $\bar{G}$ coincide with those of $G$.

A further easy consequence of (8.6) is

Corollary 8.8. Let $\Psi_{M} \in S$ be the polynomial defined after Theorem 2.8, and let $\bar{\Psi}_{M}$ be the element of $\bar{S}$ defined in analogous fashion for $M$ as $\bar{G}$-module. Then $\tau\left(\bar{\Psi}_{M}\right) \doteq \Psi_{M}$.

\subsection{Decomposition of $\bar{G}$ into graded components}

The vector space $E$ is graded, and $\bar{G}$ preserves degrees. Therefore there is a natural decomposition of $\bar{G}$ into components $\bar{G}_{i}$. In this subsection we relate the various invariants and constants we have discussed for $\bar{G}$ to those of the $\bar{G}_{i}$.

Accordingly, write $E=E_{1} \oplus E_{2} \oplus \cdots$ for the decomposition of $E$ into its graded components, with $E_{i}$ having degree $i$ (so that $E_{i}=0$ for all but finitely many $i$ ). Let $\bar{G}_{i}$ be the image of $\bar{G}$ in $\operatorname{GL}\left(E_{i}\right)$. Since $\bar{G}$ is generated 
by reflections in $E$, the groups $\bar{G}_{i}$ are generated by reflections in $E_{i}$ and we have $\bar{G}=\bar{G}_{1} \times \bar{G}_{2} \times \cdots$. Let $\bar{S}_{\bar{G}_{i}}$ be the algebra of coinvariants of $\bar{G}_{i}$ acting on $E_{i}$. If $E_{i}=0$ we take $\bar{G}_{i}=1$ and $\bar{S}_{\bar{G}_{i}}=K$. Then

$$
\bar{S}_{\bar{G}} \simeq \bar{S}_{\bar{G}_{1}} \otimes \bar{S}_{\bar{G}_{2}} \otimes \cdots
$$

We begin this subsection with the following observation which relates eigenvectors for cosets of $G$ to those for $\bar{G}$ and the $\bar{G}_{i}$. Take $\gamma \in \Gamma$ and denote by $\bar{\gamma}_{i}$ its image in $\operatorname{GL}\left(E_{i}\right)$, so that $\bar{\gamma}=\left(\bar{\gamma}_{1}, \bar{\gamma}_{2}, \ldots\right)$.

Proposition 8.9. Suppose $\gamma$ has an eigenvector $v$ such that $\gamma v=\zeta v$ for some $\zeta \in K^{\times}$. Let $\bar{v}$ denote the image of $v$ in $V / L$ and write $\bar{v}=$ $\bar{v}_{1} \oplus \bar{v}_{2} \oplus \cdots$ with $\bar{v}_{i} \in E_{i}$ (recall that $\tau$ defines an embedding of $V / L$ into E). Then for each $i, \bar{\gamma}_{i}\left(\bar{v}_{i}\right)=\zeta^{i} \bar{v}_{i}$.

Proof. Let $\left(Q_{1}, \ldots, Q_{s}\right)$ be a homogeneous basis of $E^{*}$ and suppose that $Q_{i}$ has degree $m_{i}$. Let $\left(e_{1}, \ldots, e_{s}\right)$ be the basis of $E$ which is dual to $\left(Q_{1}, \ldots, Q_{s}\right)$ and let $\pi: V \rightarrow V / L \hookrightarrow E$ be the natural morphism. Then, by definition,

$$
\bar{v}=\pi(v)=\sum_{i=1}^{s} Q_{i}(v) e_{i} .
$$

Then $\bar{\gamma}(\pi(v))=\pi(\gamma(v))=\pi(\zeta v)$. So

$$
\bar{\gamma}(\pi(v))=\sum_{i=1}^{s} \zeta^{m_{i}} Q_{i}(v) e_{i}
$$

as required.

The next statement deals with the question of regularity.

Proposition 8.10. Assume further in (8.9), that $v$ is $G$-regular. Then $\bar{v}$ is regular for $\bar{G}$, and a fortiori $\bar{v}_{i}$ is regular for $\bar{G}_{i}$, for each $i$. Thus if $\zeta \in K^{\times}$is regular for $\gamma G, \zeta^{i}$ is regular for $\bar{\gamma}_{i} \bar{G}_{i}$

Proof. For any polynomial $Q \in \bar{S}=S\left(E^{*}\right)$ and element $w \in V$, we have

$$
\tau(Q)(w)=Q(\bar{w})
$$

where $\bar{w}$ denotes the image in $E$ of the $L$-orbit of $w$. Applying (8.8), it follows that for any $\bar{G}$-module $M, \bar{\Psi}_{M}(\bar{v})=\Psi_{M}(v) \neq 0$, since $\Psi_{M}$ is always a product of linear forms corresponding to the hyperplanes of $G$, and $v$ is $G$-regular. In particular, this applies to the representation $E$, which proves that $\bar{v}$ is regular. The other statements are clear. 
Remark 8.11. The untwisted part of (8.10) is easily deduced from [BBR, Theorem 3.12 (iii)], while an untwisted analogue of (8.9) was stated without proof in [BBR, note added in proof].

We finish by relating the $M$-degrees and constants of $\bar{G}$ and those of the $\bar{G}_{i}$. Let $\bar{\Gamma}_{i}$ denote the image of $\Gamma$ in $\operatorname{GL}\left(E_{i}\right)$. Then $\langle\bar{G}, \bar{\Gamma}\rangle$ is a subgroup of $\left\langle\bar{G}_{1}, \bar{\Gamma}_{1}\right\rangle \times\left\langle\bar{G}_{2}, \bar{\Gamma}_{2}\right\rangle \times \ldots$. For each $i$, let $\bar{M}_{i}$ be a $\left\langle\bar{G}_{i}, \bar{\Gamma}_{i}\right\rangle$-module and take the $\langle\bar{G}, \bar{\Gamma}\rangle$-module $M$ to be $M=\bar{M}_{1} \otimes \bar{M}_{2} \otimes \cdots$. Then, by (8.5), we have an isomorphism of $\Gamma$-modules

$$
\left(S_{G} \otimes M\right)^{G} \simeq\left(\bar{S}_{\bar{G}_{1}} \otimes \bar{M}_{1}\right)^{\bar{G}_{1}} \otimes\left(\bar{S}_{\bar{G}_{2}} \otimes \bar{M}_{2}\right)^{\bar{G}_{2}} \otimes \cdots
$$

Now define the fake $\gamma$-degree $F_{M, \gamma}(t)$ of $M$ as the polynomial

$$
F_{M, \gamma}(t)=\sum_{i \geq 0} \operatorname{Trace}\left(\gamma,\left(\left(S_{G}\right)_{i} \otimes M^{*}\right)^{G}\right) t^{i}=\sum_{\iota \in \mathcal{B}(M, \gamma)} \varepsilon_{\iota}(M, \gamma) t^{m_{\iota}}
$$

Proposition 8.13. We have

$$
F_{M, \gamma}(t)=\prod_{i \geq 0} F_{\bar{M}_{i}, \bar{\gamma}_{i}}\left(t^{i}\right)
$$

Proof. It is clear from (8.12) that we may take $\mathcal{B}(M, \gamma)=\mathcal{B}\left(\bar{M}_{1}, \bar{\gamma}_{1}\right) \times$ $\mathcal{B}\left(\bar{M}_{2}, \bar{\gamma}_{2}\right) \times \cdots$. If $\beta_{i} \in \mathcal{B}\left(\bar{M}_{i}, \bar{\gamma}_{i}\right)(i=1,2, \ldots)$ and $\iota=\beta_{1} \otimes \beta_{2} \otimes \cdots$, then

$$
\varepsilon_{\iota}(M, \gamma) t^{m_{\iota}}=\prod_{i} \varepsilon_{\beta_{i}}\left(\bar{M}_{i}, \bar{\gamma}_{i}\right) t^{i m_{\beta_{i}}}
$$

from which the statement is clear.

This last result is a twisted version of the assertion made without proof in [BBR, note added in proof], which corresponds to the case $\gamma=\mathrm{Id}$.

\section{Appendix 1; A list of reflection cosets}

In this section, we shall classify the reflection cosets $G \gamma$ where $G$ is irreducible and $\gamma$ induces a non-inner automorphism of $G$ (up to multiplication by scalars) and regular eigenvalues for our choices of $\gamma$. The list of reflection cosets as above is given in [BMM, 3.13]. The result is given in the table concluding this article. The table in $[\mathrm{BMM}]$ gives the image of $G \gamma$ in the group of outer automorphisms of $G$, which describes the coset up to a 
scalar. In each case we choose a specific representative. First, for each natural number $d$, we choose a primitive $d^{\text {th }}$-root of unity $\zeta_{d}$. We also assume that $\zeta_{d e}^{e}=\zeta_{d}$ for every $d$, e. Before giving the table, we explain our conventions and explain how we get the numerical results. First, $o(\zeta)$ denotes the order of $\zeta$. Except for the first two examples in the table, the degrees and codegrees are given in increasing and decreasing order respectively.

A product formula. (cf. [CHEVIE]) The formula

$$
\prod_{g \in G} \operatorname{det}(1-T g \gamma)=\prod_{i}\left(1-\varepsilon_{i} T^{d_{i}}\right)^{|G| / d_{i}}
$$

is deduced from the case $M=V$ of (3.1) in the same way that (1.9) is deduced from (1.8) in $[\mathrm{Br}]$. It is used in the CHEVIE package to compute the $\varepsilon_{i}$ 's.

The imprimitive groups. Let $d$ and $e$ be two natural numbers. Let $\mu_{d}$ denote the group of $d$-th roots of unity in $K$. We choose a basis $v_{i}$ of $V$ such that the group $G(d e, e, r)$ is realized as the group of monomial matrices with non-zero entries in $\mu_{e d}$ of which the product of the non-zero entries lies in $\mu_{d}$. The automorphism $\gamma$ is induced by the diagonal matrix with diagonal entries $\left(\zeta_{e^{\prime} d}, 1, \ldots, 1\right)$, where $e^{\prime}$ divides $e$.

If $\left\{X_{i}\right\}$ is the basis of $V^{*}$ dual to $\left\{v_{i}\right\}$, the invariants of $G(d e, e, r)$ are $P_{k}=\sum_{j_{1}<\cdots<j_{k}} X_{j_{1}}^{d e} \cdots X_{j_{k}}^{d e}$ for $k=1, \ldots, r-1$ and $P_{r}=\left(X_{1} \cdots X_{r}\right)^{d}$. The corresponding degrees are $e d, 2 e d, \ldots,(r-1) e d$ and $r d$ and the corresponding $\varepsilon_{i}$ are $1, \ldots, 1$ and $\zeta_{e^{\prime}}^{-1}$.

The case $d>1$. Let us determine the $\varepsilon_{i}^{*}$ when $G=G(d e, e, r)$ with $d>1$ and $e>1$. The codegrees are $0, d e, \ldots,(r-1) d e$. According to [OT, B.1 $\left.\left(2^{\prime}\right)\right]$ one may choose as a basis of $(S \otimes V)^{G}$ the vectors $\theta_{i}=$ $\sum_{j=1}^{r} X_{j}^{(i-1) d e+1} \otimes v_{j}$. This basis is $\gamma$-invariant for our choice of $\gamma$, so we get $\varepsilon_{i}^{*}=1$. We find that $\zeta$ is regular when $\zeta^{r d}=\zeta_{e^{\prime}}$.

The case $d=1$. The group $G(e, e, r)$ is well-generated. Its codegrees are $0, e, \ldots,(r-2) e$ and $(r-1) e-r$. We may exclude the cases $e=1$ where $\gamma$ is inner and $e=r=2$ which is a non-irreducible group, so we have $r<(r-1) e$ thus the largest degree is $(r-1) e$. We may thus use the relation $\varepsilon_{i} \varepsilon_{\sigma(i)}^{*}=\varepsilon_{r}=1$ to determine the $\varepsilon_{i}^{*}$ which are, ordering the codegrees as above, $1, \ldots, 1$ and $\zeta_{e^{\prime}}$. We find that $\zeta$ is regular whenever $\zeta^{(r-1) e}=1$ or $\zeta^{r}=\zeta_{e^{\prime}}$. Note that $\gamma$ is 1-regular. 
The case ${ }^{3} G(4,2,2)$. Let $\gamma$ be a reflection in $G_{6}$ of order 3 and assume here that $G=G(4,2,2)$ embedded in $G_{6}$ as a normal subgroup of index 3. We have $d_{1}=d_{2}=4$ and, since $\gamma$ acts as a reflection on $Y$ (see the proof of Proposition 7.7), we have $\left(\varepsilon_{1}, \varepsilon_{2}\right)=\left(1, \zeta_{3}\right)$ or $\left(1, \zeta_{3}^{2}\right)$. If we take

$$
\gamma=\frac{\zeta_{4}+1}{2 \zeta_{3}}\left(\begin{array}{cc}
-1 & 1 \\
\zeta_{4} & \zeta_{4}
\end{array}\right)
$$

Then $\operatorname{det} \gamma=\zeta_{3}, \gamma$ stabilises the vector space $S_{4}^{G}$ which is generated by $P_{1}=X_{1}^{4}+X_{2}^{4}$ and $P_{2}=X_{1}^{2} X_{2}^{2}$. An easy computation shows that $\left(\varepsilon_{1}, \varepsilon_{2}\right)=\left(1, \zeta_{3}\right)$. Note that $\gamma$ permutes cyclically the generators $\left(\begin{array}{cc}-1 & 0 \\ 0 & 1\end{array}\right)$, $\left(\begin{array}{cc}0 & -\zeta_{4} \\ \zeta_{4} & 0\end{array}\right)$ and $\left(\begin{array}{ll}0 & 1 \\ 1 & 0\end{array}\right)$ of $G$.

On the other hand, $\left(d_{1}^{*}, d_{2}^{*}\right)=(0,4)$ and, since $\gamma$ is 1-regular (by direct check), we find that $\varepsilon_{1}^{*}=1$ and $\varepsilon_{2}^{*}=\zeta_{3}^{-1}$. Now, $\zeta$ is a regular eigenvalue for $G \gamma$ if and only if $\zeta^{4}=1$.

The case ${ }^{2} \boldsymbol{G}_{\boldsymbol{7}}$. Note that $\gamma$ comes from the normal embedding $G_{7} \subset$ $G_{15}$. We choose $\gamma$ to be a reflection of order 2. It acts as a reflection on $Y$. So, the pairs $\left(d_{i}, \varepsilon_{i}\right)$ are $(12,1),(12,-1)$. A direct check shows that the chosen $\gamma$ is 1-regular, whence the $\left(d_{i}^{*}, \varepsilon_{i}^{*}\right)$ must be $(0,1),(12,-1)$. An eigenvalue $\zeta$ is regular if and only if $\zeta^{12}=1$.

The cases ${ }^{2} \boldsymbol{F}_{\mathbf{4}}$ and ${ }^{\mathbf{3}} \boldsymbol{D}_{\mathbf{4}}$. These are Coxeter groups. One may choose $\gamma$ as a diagram automorphism which is 1-regular, then the $\varepsilon_{i}$ are determined from $\gamma^{\prime}$ 's eigenvalues on $V$. The $\varepsilon_{i}^{*}$ are equal to the $\varepsilon_{i}$ if we order the codegrees in increasing order as well as the degrees.

For ${ }^{3} D_{4}$ the pairs $\left(d_{i}, \varepsilon_{i}\right)$ are $(2,1),\left(4, \zeta_{3}\right),\left(4, \zeta_{3}^{2}\right),(6,1)$ where $\zeta_{3}$ is a primitive cubic root of unity, and the pairs $\left(d_{i}^{*}, \varepsilon_{i}^{*}\right)$ are $(0,1),\left(2, \zeta_{3}\right),\left(2, \zeta_{3}^{2}\right)$, $(4,1)$. An eigenvalue $\zeta$ is regular if and only if it has order $1,2,3,6$ or 12 .

For ${ }^{2} F_{4}$ the pairs $\left(d_{i}, \varepsilon_{i}\right)$ are $(2,1),(6,-1),(8,1),(12,-1)$ and the pairs $\left(d_{i}^{*}, \varepsilon_{i}^{*}\right)$ are $(0,1),(4,-1),(6,1),(10,-1)$. An eigenvalue $\zeta$ is regular if and only if it has order $1,2,4,8,12$ or 24 .

The case ${ }^{4} G(3,3,3)$. In the basis as above for the imprimitive groups, we may choose $\gamma=\frac{-1}{\sqrt{-3}}\left(\begin{array}{ccc}\zeta_{3} & 1 & \zeta_{3}^{2} \\ 1 & 1 & 1 \\ \zeta_{3}^{2} & 1 & \zeta_{3}\end{array}\right)$. It is of order 4 , and does not stabilize any set of generators of $G=G(3,3,3)$ of cardinality 3 . We find 
that the pairs $\left(d_{i}, \varepsilon_{i}\right)$ are $\left(3, \zeta_{4}\right),\left(3,-\zeta_{4}\right),(6,1)$. Since the group is wellgenerated we deduce that the pairs $\left(d_{i}^{*}, \varepsilon_{i}^{*}\right)$ are $(0,1),\left(3, \zeta_{4}\right),\left(3,-\zeta_{4}\right)$. An eigenvalue $\zeta$ is regular if and only if $\zeta^{6}=1$.

The case ${ }^{2} \boldsymbol{G}(\mathbf{3}, \mathbf{3}, \mathbf{3})$. Take for $\gamma$ the square of the above matrix. Then the new $\varepsilon_{i}$ are the squares of the previous ones and similarly for the $\varepsilon_{i}^{*}$. Again, $\zeta$ is regular if and only if $\zeta^{6}=1$.

The case ${ }^{\mathbf{2}} \boldsymbol{G}_{\mathbf{5}}$. Let $\gamma$ be a reflection of order 2 in $G_{14}$ which does not lie in $G_{5}$ (which is a normal subgroup of $G_{14}$ of index 2). Again, $\gamma$ acts as a reflection of order 2 on $Y$. Since the degrees of $G_{5}$ are $(6,12)$ and those of $G_{14}$ are $(6,24)$, we get that $\varepsilon_{1}=1$ and $\varepsilon_{2}=-1$. As $G$ is well-generated, we may deduce that the pairs $\left(d_{i}^{*}, \varepsilon_{i}^{*}\right)$ are $(0,1),(6,-1)$. We find that $\zeta$ is regular if and only if it is of order $1,2,3,6,8$ or 24 .

To obtain the above statement, one may choose as generators of $G_{5}$ the elements $s_{+}$and $s_{-}$, where $s_{\varepsilon}=\frac{1}{2}\left(\begin{array}{cc}(-1+\sqrt{-2}) \zeta_{3} & \varepsilon \zeta_{12} \\ \varepsilon \zeta_{12} & (-1-\sqrt{-2}) \zeta_{3}\end{array}\right)$ for $\varepsilon \in\{+,-\}$. Then, one may take $\gamma=\left(\begin{array}{cc}1 & 0 \\ 0 & -1\end{array}\right)$. With these choices, $\gamma$ interchanges $s_{+}$and $s_{-}$.

\section{Appendix 2; Proofs of (2.8) and (2.11)}

Proof of Theorem 2.8. Let $\Psi_{M}=\prod_{H \in \mathcal{A}} L_{H}^{N_{H}(M)}$ and let $\Psi_{M}^{\prime}$ be the element of $S$ defined by $\prod_{\iota \in \mathcal{B}(M)} \iota=\Psi_{M}^{\prime} \otimes\left(y_{1} \wedge \cdots \wedge y_{m}\right)$. Observe that

$$
\Psi_{M \oplus M^{\prime}}=\Psi_{M} \Psi_{M^{\prime}} \quad \text { and } \quad \Psi_{M \oplus M^{\prime}}^{\prime}=\Psi_{M}^{\prime} \Psi_{M^{\prime}}^{\prime}
$$

Now, let us first assume that $\mathcal{A}(G)=\{H\}$, so that $G=G_{H}$. By (8.14), the Theorem need only be checked when $M$ is irreducible, i.e. affords the character $\operatorname{det}^{i}$ for some $i \in\{0,1, \ldots, e-1\}$, where $e=|G|$. Since here $\mathcal{H}$ has basis the set of $L_{H}^{i}$, this case is clear.

Now, consider the general case. Let $\left(u_{1}, \ldots, u_{m}\right)$ be a basis of $(\mathcal{H} \otimes$ $\left.M^{*}\right)^{G}$. Write $u_{i}=\sum_{j=1}^{m} q_{j i} \otimes y_{j}$, with $q_{j i} \in S$. Then $u_{1} \cdots u_{m}=\operatorname{det}\left(q_{i j}\right)$. $\left(y_{1} \wedge \cdots \wedge y_{m}\right)$, so that $\Psi_{M}^{\prime}=\operatorname{det} q_{i j}$. Now for every $H \in \mathcal{A}(G), u_{i} \in$ $\left(S \otimes M^{*}\right)^{G} \subset\left(S \otimes M^{*}\right)^{G_{H}}$. Hence if we express $u_{i}$ as a linear combination of elements of an $S^{G_{H}}$-basis of $\left(S \otimes M^{*}\right)^{G_{H}}$ with coefficients in $S^{G_{H}}$, we see that $\Psi_{M}^{\prime}$ is divisible by its analogue for $G_{H}$, which $\doteq L_{H}^{N_{H}(M)}$ by the previous discussion. Since the distinct $L_{H}$ are pairwise coprime, it follows that $\Psi_{M}$ divides $\Psi_{M}^{\prime}$. It therefore suffices to show that $\Psi_{M}^{\prime}$ is non-zero, and has degree $\sum_{H \in \mathcal{A}(G)} N_{H}(M)$. 


\begin{tabular}{|c|c|c|c|c|c|c|c|c|c|}
\hline 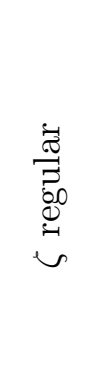 & $\begin{array}{l}j \\
\| \\
i \\
\vdots\end{array}$ & 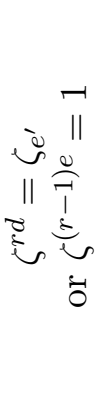 & $\begin{array}{l}-11 \\
i n\end{array}$ & $\begin{array}{l}-1 \\
i \\
i\end{array}$ &  & 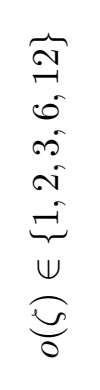 & 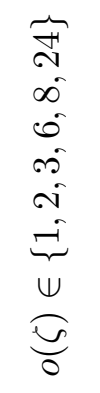 & $\begin{array}{l}\underset{11}{\sigma} \\
\stackrel{\sim}{\pi}\end{array}$ & 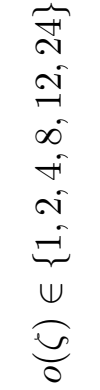 \\
\hline $\begin{array}{c}\sigma^{*}{ }^{*} \omega^{*} \\
\vdots \\
\vdots \\
\vdots \\
\sigma^{*}-{ }^{*} \\
\omega^{*}\end{array}$ &  & 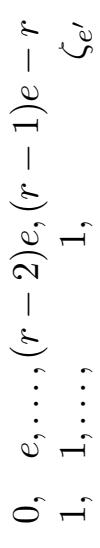 & $\begin{array}{l}\infty \vec{j} \\
\infty \\
\infty \\
\infty\end{array}$ & $\begin{array}{c}\infty \\
\infty \\
\infty \\
\infty \\
0\end{array}$ & $\begin{array}{l}+1-1 \\
0-1\end{array}$ &  & $\begin{array}{c}0 \multimap \\
0-i\end{array}$ & $\begin{array}{l}\stackrel{\sim}{-} \text { i } \\
\sigma-i\end{array}$ & 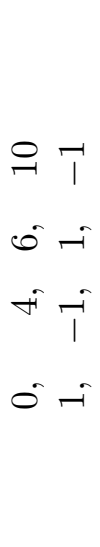 \\
\hline 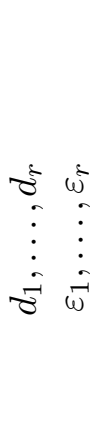 & 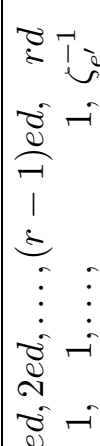 & 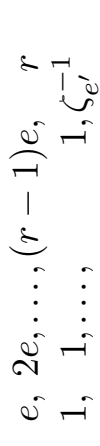 &  & $\begin{array}{l}0-1 \\
+i-i \\
+i-i\end{array}$ & $\begin{array}{l}+\infty \\
+\infty\end{array}$ & 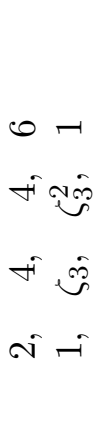 & $\begin{array}{l}\text { I } \\
0 \sim\end{array}$ & $\begin{array}{l}\stackrel{\sim}{\sim} \text { I } \\
\stackrel{\sim}{\sim}-i\end{array}$ &  \\
\hline छे &  & $\begin{array}{c}2 \\
0 \\
0 \\
0 \\
0\end{array}$ & $\underset{\sigma}{\stackrel{\infty}{\infty}}$ & $\underset{\sim}{\stackrel{m}{\infty}}$ & $\begin{array}{l}\tilde{N} \\
\text { v } \\
\underset{d}{J}\end{array}$ & $\overbrace{\infty}^{+H}$ & $\overbrace{N}^{10}$ & $\overbrace{N}^{N}$ & $\underset{N}{T^{H}}$ \\
\hline
\end{tabular}


For the first statement, we prove that if $v \in V$ is such that $C_{G}(v)=1$, where $C_{G}(v)=\{x \in G \mid x(v)=v\}$, then $\Psi_{M}^{\prime}(v) \neq 0$. Note that for any non-trivial element $x \in G, V_{x}:=\{v \in V \mid x(v)=v\}$ is a subspace of positive codimension in $V$, whence $V \backslash \bigcup_{x \in G, x \neq \operatorname{Id}_{V}} V_{x} \neq \emptyset$, so that such elements $v$ exist. Given one, if $G v$ is its $G$-orbit, then the map $G \rightarrow G v$ defined by $g \mapsto$ $g(v)$ is bijective. Let $\mathcal{F}$ be the space of functions $G v \rightarrow K$, endowed with its natural $G$-module structure. The restriction map $S \rightarrow \mathcal{F}$ is evidently surjective. Since elements of $S^{G}$ are constant on $G v$, the restriction map $\mathcal{H} \rightarrow \mathcal{F}$ is also surjective, and by dimension, is an isomorphism of $G$ modules. Let $f \in \mathcal{H}$ be such that $f(v)=1$ and $f(g(v))=0$ if $g \neq \operatorname{Id}_{V}$. Consider the map $\nu: \mathcal{H} \otimes M^{*} \rightarrow M^{*}$, given by $h \otimes x \mapsto h(v) x$. The restriction of $\nu:\left(\mathcal{H} \otimes M^{*}\right)^{G} \rightarrow M^{*}$ is an isomorphism of $K$-vector spaces, since by dimension, it suffices to show that it is surjective, which easily follows from the fact that $\nu\left(\sum_{g \in G}{ }^{g} f \otimes{ }^{g} x\right)=x$ for every $x \in M^{*}$. But the matrix of $\nu$ with respect to the bases $\left(u_{1}, \ldots, u_{m}\right)$ and $\left(y_{1}, \ldots, y_{m}\right)$ is exactly $q_{j i}(v)$, whose determinant is $\Psi_{M}^{\prime}(v)$; it follows that $\Psi_{M}^{\prime}$ is non-zero.

It remains to show that $N(M)=\sum_{H \in \mathcal{A}(G)} N_{H}(M)$. Define the fake degree $F_{M}(t)$ of $M$ as the Poincaré polynomial $\sum_{\iota \in \mathcal{B}(M)} t^{m_{\iota}}$ of $\left(\mathcal{H} \otimes M^{*}\right)^{G}$, where $t$ is an indeterminate. It is then clear that $N(M)=\left.\frac{\partial F_{M}}{\partial t}\right|_{t=1}$. However from Molien's formula, if $\chi$ is the character of the $G$-module $M$, we have $F_{M}(t)=\frac{\prod_{\iota \in \mathcal{B}(V)}\left(1-t^{d_{\iota}}\right)}{|G|} \sum_{g \in G} \frac{\chi(g)}{\operatorname{det}_{V}(1-g t)}$. Taking derivatives, we see that the terms where $g$ is not 1 or a reflection do not contribute to $N(M)$. To sum the remaining terms, we use the fact that the number $|\operatorname{Ref}(G)|$ of reflections of $G$ is equal to $\sum_{\iota}\left(d_{\iota}-1\right)$ to obtain $N(M)=$ $\chi(1)|\operatorname{Ref}(G)| / 2+\sum_{s \in \operatorname{Ref}(G)} \frac{\chi(s)}{\operatorname{det}(s \mid V)-1}$. This expression is exactly the sum of the corresponding expressions for $G_{H}$, over all $H \in \mathcal{A}(G)$, whence the result.

Proof of Lemma 2.11. Let $M$ be a $G$-module of dimension $m$ such that any reflection of $G$ acts as a reflection in $M$. Then for $H \in \mathcal{A}$, we have in the notation preceding the statement of Theorem 2.8, the $G_{H \text {-module }}$ decomposition $M^{*}=\bigoplus_{i=1}^{m} \xi_{H}^{e_{i}}$, where $e_{1} \neq 0$ and $e_{i}=0$ for $i>1$. Then $\Lambda^{m} M^{*}=\xi_{H}^{e_{1}}$, and $N_{H}(M)=N_{H}\left(\Lambda^{m} M\right)=e_{1}$. Since this holds for any $H \in \mathcal{A}$, and $N(M)=\sum_{H \in \mathcal{A}} N_{H}(M)$ for any $M$, the Lemma follows.

\section{REFERENCES}

[B] D. Bessis, Zariski theorems and diagrams for braid groups, Inv. Math., 145 (2001), 487-507. 
[B2] D. Bessis, Topology of complex reflection arrangements, math.GT/0411645, to appear.

[BBR] D. Bessis, C. Bonnafé and R. Rouquier, Quotients et extensions de groupes de réflexion, Math. Ann., 323 (2002), 405-436.

[BL] J. Blair and G. I. Lehrer, Cohomology actions and centralisers in unitary reflection groups, Proc. Lond. Math. Soc. (3), 83 (2001), 582-604.

[Br] M. Broué, Reflection groups, braid groups, Hecke algebras, finite reductive groups, Current developments in mathematics, 2000, Harvard university \& M.I.T. international press, Boston (2001), pp. 1-103.

[BMM] M. Broué, G. Malle and J. Michel, Towards Spetses I, Transf. Groups, 4 (1999), $157-218$.

[CHEVIE] see www.math.jussieu.fr/ ${ }^{j m i c h e l / c h e v i e . ~}$

[L] G. I. Lehrer, A new proof of Steinberg's fixed-point theorem, Int. Math. Res. Not., 28 (2004), 1407-1411.

[LM] G. I. Lehrer and J. Michel, Invariant theory and eigenspaces for unitary reflection groups, C.R.A.S., 336 (2003), 795-800.

[LS1] G. I. Lehrer and T. A. Springer, Intersection multiplicities and reflection subquotients of unitary reflection groups I, Geometric group theory down under (Canberra, 1996), de Gruyter, Berlin (1999), pp. 181-193.

[LS2] G. I. Lehrer and T. A. Springer, Reflection subquotients of unitary reflection groups, Canad. J. Math., 51 (1999), 1175-1193.

[Ma] G. Malle, Splitting fields for extended complex reflection groups and Hecke algebras, Transformation groups, to appear.

[MN] H. Morita and T. Nakajima, The coinvariant algebra of the symmetric group as a direct sum of induced modules, Osaka J. Math., 42 (2005), 217-231.

[OS] P. Orlik and L. Solomon, Unitary reflection groups and cohomology, Inv. Math., 59 (1980), 77-94.

[OT] P. Orlik and H. Terao, Arrangements of hyperplanes, Grunlehren der math. Wissenschaften 300, Springer-Verlag, 1991.

[Sp] T. Springer, Regular elements of finite reflection groups, Invent. Math., 25 (1974), 159-198.

[Ste] J. R. Stembridge, On the eigenvalues of representations of reflection groups and wreath products, Pacific J. Math., 140 (1989), 353-396.

C. Bonnafé

UFR ST

Laboratoire de Mathématiques de Besançon

CNRS (UMR 6623)

16 Route de Gray

25000 Besançon

France

bonnafe@math.univ-fcomte.fr 
G. I. Lehrer

School of Mathematics and Statistics

University of Sydney

Sydney, NSW 2006

Australia

gusl@maths.usyd.edu.au

J. Michel

Institut de Mathématiques

Université Paris VII

175 rue du Chevaleret

75013 Paris

France

jmichel@math.jussieu.fr 